\title{
2 Imperfektion
}

\section{Strategien und Wirkungsweisen der nicht perfekten Rede}

\subsection{Einführung}

Das vorangehende Kapitel hat uns diverse Möglichkeiten, Modi und Wirkungsweisen der Elaboration aus der klassischen Rhetorik für die Rede aufgezeigt, die immer noch Gültigkeit haben und sich auch auf andere Téchnai anwenden lassen. Nicht mehr so klar scheint in den Gebieten, die wir heute als Künste bezeichnen, jedoch die strikte Ausrichtung an den Idealen der Perfektion, Sorgfalt und Harmonie zu sein, die für die Ausarbeitung der Rede in der Antike noch uneingeschränkt Geltung hatten und nach denen Redner bedingungslos streben sollten. Während Technik und Handwerk bis heute einem Präzisions- und Vollkommenheits-Ideal verpflichtet geblieben sind, hat sich die Kunst von ihrem Regelkorsett befreit, ja zeichnet sich oftmals gerade durch ein Abweichen und Hinterfragen, einen spielerischen Umgang oder ein rebellisches Brechen produktionstechnischer und stilistischer Normen aus: Malerei und Zeichnung können flüchtig, ungelenk oder rasch hingeworfen statt sorgfältig ausgearbeitet sein. Ein literarischer Text muss nicht harmonisch sein, sondern kann ebenso gut stockend, rissig oder abgehackt geschrieben sein. Skulpturen werden mitunter grob, rau oder zerkratzt belassen, anstatt fein ausgeschliffen zu werden. Kunstfotografien wirken bewusst schrill oder hässlich und nicht grazil oder elegant und Plakate können übertrieben und disharmonisch ausgestaltet sein anstatt ausgewogen. Entsprechend ist auch die Person der Künstlerin oder des Gestalters kaum mehr an Ideale wie Gewissenhaftigkeit, Sorgfalt oder Fleiß gebunden, sondern wird allenfalls an Anforderungen des persönlichen Ausdrucks oder (als Ideale vielleicht auch schon wieder überholt) der Genialität oder Kreativität gemessen. Der Gedanke der «künstlerischen Freiheit` ist heute fast schon zu einem Gemeinplatz geworden und auch im Design gibt es bis heute Positionen, die den «Mythos des genialen Künstler-Entwerfers» pflegen, der sich frei von allen Restriktionen entfaltet (Schneider 2005: 35). Grammatik scheint heutzutage etwas zu sein, das wir in der Schule lernen, während echte Literatur sie nach Belieben ignoriert. Präzise Maltechnik und akkurater Farbauftrag mögen wichtig sein für Anfänger, erst der improvisierte und individuelle Umgang mit Pinsel und Farbe macht jedoch die Kunst aus. Im Grafikdesign bilden technische Fertigkeiten und die Beherrschung der Layout-Software sicherlich eine notwendige Basis, doch auch hier zeigt sich wahre Könnerschaft oftmals gerade im Regelbruch. Man könnte den Einsatz der Imperfektion in der heutigen Kunst und Gestaltung auch als Widerstand gegen ein streberhaft und fade empfundenes Perfektionierungsdenken verstehen. 
Ist dieses Widerspenstige der Kunst, dieser Vorbehalt gegenüber dem Vollkommenen und Schönen etwas ganz Neues, Modernes? In seiner kritischen Radikalität auf jeden Fall. Nichtsdestoweniger wird sich im Folgenden zeigen, dass bereits der klassischen Rhetorik eine gewisse Widerständigkeit eingebaut war, die den absoluten Perfektionswillen der Redekunst in Frage stellt. Schon im vorherigen Kapitel wurden ja die Grenzen des Ausfeilens und Schleifens am eigenen Werk sichtbar, da dieses Streben leicht in Übereifer oder Künstlichkeit ausarten kann. Außerdem wurde aufgezeigt, dass in der Antike die niedrige Stilhöhe als eigenständiger Elaborationstypus anerkannt war, der auf verschiedenen Ebenen bewusst unelaboriert, beinahe umgangssprachlich bleibt. Mit dem Konzept der rhetorischen Figur hat die Rhetorik vielleicht sogar den Prototypen des kunstvollen Regelbruchs beschrieben. Denn in gewissem Sinn sind die Figuren, Schemata oder Tropen selbst Abweichungen oder zumindest Spielarten der geltenden Sprachnorm: Figuren verstoßen oftmals gegen die Regeln der Komposition oder der Grammatik oder sie umgehen gewitzt die Art und Weise, wie man Dinge normalerweise ausdrücken würde. Vitium wird zu Virtus, der eigentliche Fehler zu einer Tugend umgedeutet. In der Literatur bieten Figuren und Tropen den Autorinnen und Autoren bis heute eine Vielzahl von Abweichungsmustern und Möglichkeiten, Sprache zu variieren, aufzulösen oder umzuformen - spielerisch oder radikal, subtil oder affektstark.

Die Redekunst als wirkungsorientierte Téchnē hat sich immer schon die Frage gestellt, wie eine Rede von ihrem Publikum aufgenommen wird und wie es Rednern gelingen kann, im jeweiligen Kontext angemessen zu sprechen. Wie können sie bei ihrem Auftritt die Zuhörer sabholen` und was müssen sie tun, um glaubwürdig und sympathisch zu erscheinen? Gerade in Fragen der Aufrichtigkeit und Authentizität kann sich eine allzu kunstvoll geschliffene Rede oder ein makellos gehaltener Vortrag geradezu nachteilig auswirken. Wer die Rhetorik perfekt beherrscht, entfernt sich unwillkürlich von der natürlichen Kommunikationspraxis, die wir im Alltag pflegen und schafft damit eine Distanz zum Publikum. Noch schlimmer: Bei einem Redner, der offensichtlich alle Regeln der Kunst beherrscht, könnte man auf die Idee kommen, dass er die Kraft seiner rhetorischen Kunst missbraucht. Führt ein Redner, der seine Worte besonders sorgfältig auswählt, nicht vielleicht etwas im Schilde? Umgekehrt erscheinen uns Personen authentisch, die frei heraus sprechen, ohne viel zu überlegen - scheinbar wie ihnen «der Schnabel gewachsen ist». Ja, es kann sogar sympathisch wirken, wenn sich ein Redner verhaspelt oder ihm die Worte fehlen. Zeigt sich Wahrhaftigkeit nicht erst dann, wenn man ins Stammeln kommt?

All diese Ausformungen der Rede - das Unvorbereitete und Hingeworfene, das Simple und Alltagssprachliche, das Stammelnde und Stotternde, das Suchende oder Um-Worte-Verlegene - zeugen von einem wenig elaborierten bis 
fehlerhaften Duktus, einer ungeschliffenen oder eben imperfekten Elaboration. Auch wenn 〈Imperfektion〉 als Terminus weder innerhalb des Lehrsystems der Rhetorik noch in den anderen Künsten detailliert beschrieben wird, ist das Imperfekte als Stil- und Wirkungsphänomen doch elementar für Künste wie die Rede, die Literatur, die bildende Kunst oder das Design. Imperfektion kann als Gradmesser der Elaboration verstanden werden, als Kontrapunkt der hoch elaborierten, geschliffenen oder perfekten Kunst: Eine imperfekte Rede ist eine wenig oder unzureichend elaborierte Rede, ein imperfekter Text ein wenig oder mangelhaft elaborierter Text, ein imperfektes Bild ein wenig oder schlecht elaboriertes Bild. Man sollte dabei zwei Aspekte der Imperfektion unterscheiden: Dass ein Werk nicht perfekt ausgearbeitet ist, lässt eine wertende und eine nicht wertende Sichtweise $\mathrm{zu}$. Im neutralen Sinn ist mit Imperfektion einfach eine niedrige Elaborationsstufe bezeichnet. Im (ab)wertenden Sinn wird mit «Imperfektion〉 dagegen ein Fehler oder Mangel in der Ausführung benannt, eine unsorgfältige, schlechte oder gegen eine geltende Norm verstoßende Elaborationsweise. In der Bedeutung des Fehlers ist Imperfektion grundsätzlich etwas, das es zu vermeiden gilt. Aus produktionstechnischer Sicht lässt sich zudem eine Unterscheidung zwischen zwei verschiedenen Operationsmodi ziehen, aus denen eine Imperfektion resultieren kann - ob nämlich die Imperfektion gezielt eingesetzt wird oder ob sie bloß unterläuft. Imperfektionen können also das Resultat einer strategischen Überlegung sein oder sie können ungewollt oder gar contrecœur auftreten. Bei strategischer Anwendung können Imperfektionen zum professionellen oder meisterhaften Repertoire einer Kunst gehören. Tritt die Imperfektion dagegen unabsichtlich auf, ist sie auf mangelnde Beherrschung der Téchnē zurückzuführen und als Form des Dilettantismus zu deuten.

Im System der klassischen Rhetorik lässt sich Imperfektion wortwörtlich als Gegenbegriff zum vorherrschenden Ideal der perfekten Ausarbeitung sehen. Somit steht hier auch der Begriff des Fehlers bzw. des Vitium als Bewertungskriterium im Zentrum. Alle Redeweisen, welche gegen die Virtutes oder Vorzüge der Rede verstoßen, sind Imperfektionen im negativen Sinn und somit zu verwerfen. Bereits im antiken System der Rhetorik findet sich jedoch nicht nur die Bedeutungsrichtung des Fehlerhaften, sondern erhalten bestimmte Formen der Imperfektion, wie etwa die bereits genannten rhetorischen Figuren, eine eigene Berechtigung und eine erstaunliche Relevanz. Werden Abweichungen von den Vorgaben der Grammatik, der Wortzusammensetzung, des harmonischen Redeflusses oder der stilistischen Geschliffenheit bewusst ausgewählt und mit Bedacht angewendet, können diese Formen der nicht perfekten Ausarbeitung zu einem legitimen Wirkungsmittel umgedeutet werden. Sogar offensichtliche Fehler werden so lizenziert. Und da gerade die Alltagssprache und die Sprechweise von Laienrednern im Vergleich zu perfekt geschliffenen Reden besonders natürlich, spontan 
und authentisch wirken, nimmt sich die klassische Redekunst - anders als dies zunächst zu erwarten wäre - sogar gewisse Formen der ungewollt imperfekten Rede zum Vorbild. Um die überraschenden Qualitäten der dilettantisch imperfekten Rede wird es später noch gehen (Kap. 3). In diesem Kapitel werden die verschiedenen Möglichkeiten der strategisch motivierten und rhetorisch legitimierten Anwendung der Imperfektion genauer erforscht, welche die klassische Rhetorik bereithält.

\subsection{Rauheit: Imperfektionen im Redefluss}

Für eine ausgefeilte Rede empfiehlt sich aus Sicht der klassischen Rhetorik ein klangvoller, harmonischer und wohlgefügter Redefluss: Alle Worte sollen am richtigen Ort sein und dem Ganzen eine ansprechende Melodie und Ausgewogenheit verleihen - so fordert es laut Cicero die Wortfügung (conlocatio) (Cic. de orat. III 171-172). Insbesondere gehe es darum, einen rauen Zusammenstoß oder ein Auseinanderklaffen der Worte $\mathrm{zu}$ vermeiden und die Rede $\mathrm{zu}$ einem fugenlosen, geschliffenen Ganzen zu formen (Cic. de orat. III 171-172). Quintilian attestiert das beste Urteil über die Rede den Ohren, die «spüren, wenn es voll klingt, reklamieren, wo noch etwas fehlt, verletzt werden durch Brüchiges, geschmeichelt durch Glattes, aufgeregt durch den Schwung des Treffenden, und die billigen, was auf festen Beinen steht, aufgreifen, was lahm wirkt, und verschmähen, was überflüssig und überhäuft ist» (Quint. inst. IX 4, 116). Wortfügungen, die den Wohlklang oder Rhythmus stören, sollten deshalb grundsätzlich vermieden werden. Durch ein Aneinanderreihen einsilbiger Wörter würde die Rede zerhackt und hüpfend erscheinen, durch allzu lange Wörter schleppend und durch allzu ähnliche Wörter langweilig (Quint. inst. IX 4, 42-43).

Gleichwohl befindet Quintilian, dass die Wortfügung im Zweifelsfall lieber hart und rau klingen solle als weichlich und kraftlos. Ein allzu ängstliches Vermeiden von Kollisionen könne «den Schwung der Rede lähmen und von Wichtigerem ablenken» (Quint. inst. IX 4, 35). Ein Redner, der den harmonischen Rhythmus allzu konsequent und offensichtlich verfolge, laufe zudem Gefahr, der «handgreiflichen Künstelei» verdächtigt zu werden, «und je lieblicher es wirkt, um so mehr verliert und zerstört seine Glaubwürdigkeit und alle gewonnenen Gefühle und Regungen, wer bei solcher gekünstelten Sorgfalt ertappt ist» (Quint. inst. IX 4, 143). Die Wahl einer harmonischen Wortfolge könne daher nicht einfach bedeuten, die Rede nach einem strikten Versmaß zu rhythmisieren. So solle der Fluss der Rede zwar nicht arhythmisch sein, was «ungebildet und bäuerisch» wirken würde, aber auch nicht vollrhythmisch wie in der Poesie (Quint. inst. IX 4, 56). Eine ähnliche Ansicht findet sich schon bei Aristoteles, der zwar einen 
Rhythmus für die Rede einfordert, der jedoch keinesfalls «peinlich genau» eingehalten werden sollte: «Die Form der Rede darf weder metrisch gebunden noch unrhythmisch sein. Ersteres wirkt ja nicht überzeugend (es erscheint nämlich gekünstelt) und lenkt zugleich ab» (Aristot. rhet. III 8, 1, 1408b).

Die Wortfügung soll gemäß der antiken Rhetorik also weder klang- und kunstlos, noch allzu auffällig elaboriert sein. Um einen natürlich «dahinströmenden > Redefluss zu erlangen, kann, wie wir gleich sehen werden, eine gezielt belassene Rauheit oder eingebaute Unregelmäßigkeit angebracht sein. Um einen spannungsvollen Klang zu erreichen, ist mitunter eine bewusste Abweichung von grammatikalischen oder kompositorischen Regeln das passende Hilfsmittel. Offensichtliche Anzeichen von Ungeschliffenheit oder sogar eigentliche Fehler können so zum Kunstgriff werden. Dass es dabei auch um ein bewusstes Verbergen der eigenen Redekunst und der damit verbundenen Wirkungsintentionen geht und dass die Abweichungsmuster, aus denen sprachliche, performative oder inhaltliche Fehler resultieren, zugleich die Quelle vieler rhetorischer Figuren sind, wird sich in diesem Teilkapitel (2.2) bereits andeuten. Beide Aspekte werden in den darauffolgenden Unterkapiteln (2.3. und 2.4) noch gesondert betrachtet werden.

\subsubsection{Ungeschliffen reden: Die Naturbelassenheit der Sprache}

Trotz der Ungeschliffenheit seiner Worte attestiert Cicero dem Rechtsgelehrten Scaevola eine große Überzeugungskraft. Der Senat habe Scaevola nämlich oft in wesentlichen Fragen zugestimmt, wenn dieser «kurz und ungeschliffen» (impolite) gesprochen habe (Cic. de orat. I 214). Die knappe und wenig ausgefeilte Rede wird aus Sicht der klassischen Rhetorik somit durchaus als wirkungsmächtig erkannt. Dies ist insofern erstaunlich, als das antike System der Rhetorik auf den Idealen der Perfektion, Geschliffenheit und Harmonie aufbaut und davon ausgeht, dass sich die Überzeugungskraft der Rede durch die Steigerung des Elaborationsgrads optimieren lässt. Ein gewisser Zwiespalt zeigt sich auch in Bezug auf die stimmliche Ausgestaltung des Vortrags. Obschon Cicero eine kraftlose, unmelodische oder misstönende Stimme bei einem Redner eigentlich klar als Mangel deutet, der von den Rednern normalweise vermieden würde, meint er zu erkennen, dass manche Redner gerade absichtlich nach diesem Fehler trachten würden: «Gewisse Leute finden an einem rohen, ungeschliffenen Organ (rustica et agrestis vox) Gefallen» (Cic. de orat. III 42). Andernorts findet sich die Bemerkung, dass das Publikum einem Redner, der heiser (raucus) sei, oftmals besonders aufmerksam zuhöre (Cic. de orat. I 259). 
Obgleich die antike Redelehre Ungeübtheit, Ungeschliffenheit und Disharmonie im Grundsatz ablehnt, hebt sie an mehreren Stellen die Kraft und das Reizvolle, mitunter auch Einzigartige nicht perfekter Sprech- und Vortragsweisen hervor. Diese gegensätzlichen Haltungen gegenüber der Imperfektion spiegeln sich in der an antiken Vorbildern orientierten Rhetorik des spanischen Humanisten Juan Luis Vives. Zur Erlangung einer seriösen Wirkung in der Rede bedürfe es, wie er sagt, einerseits gewichtiger Sentenzen, solider Argumente und eines robusten und sicheren Klangs der Stimme. Andererseits könne die Seriosität gerade durch den gelegentlichen Einsatz von Rauheit (asperitas), durch ein Zögern (impedimentum) oder gar Anhalten (sufflaminatio) im Redefluss verstärkt werden (Vives 1536: II, 142). Cicero meint bei Rednern, welche in einer Stilart brillierten, neben Vertretern, die ein ausgefeiltes Verfahren wählten, stets auch solche zu erkennen, die eine raue oder ungeschliffene Redeweise bevorzugten. Die Kraft und Eindringlichkeit des erhabenen Stils «suchten die einen mit einer rauhen, unfreundlichen, harten Redeweise, ohne Rundung und rhythmischen Schluß der Periode zu erreichen, die anderen mit einer glatten, geordneten und periodisch kadenzierenden Art zu sprechen» (Cic. orat. 6, 20). Auch beim schlichten Stil gebe es jene gewieft vorgehenden Redner, «die ungefeilt [impoliti] sprechen und vorsätzlich den Ungebildeten und Ungeschulten ähneln», neben solchen, die zwar nüchtern, aber mit feinerer Klinge (concinniores) an die Arbeit gingen (Cic. orat. 6,20$)$.

Ein geübter Redner dürfte deshalb ein ausgewogenes Mischungsverhältnis suchen zwischen der nach allen Regeln der Kunst elaborierten Rede, die eloquent und selbstbewusst vorgetragen wird, und einem ungeschliffen und natürlich wirkenden, auch mal ins Stocken oder Zögern kommenden Redefluss. Eine solche Mischung wirkt unter Umständen kraftvoller, ungekünstelter, sympathischer und sogar glaubwürdiger als eine stark aufpolierte Rede. Wird die Sprache, die für eine Rede gewählt wird, bewusst roh belassen, so kommt dies im Grunde einem Trick gleich, auf den schon Aristoteles hinweist: «Daher muss der Redner unauffällig ans Werk gehen und keinen gekünstelten, sondern einen natürlichen Eindruck erwecken» (Aristot. rhet. III 2, 19, 1404b). Die unvermeidbare Künstlichkeit, die durch die Ausarbeitung einer Rede entsteht, lasse sich clever verbergen, indem man sich bei der Komposition des Redetexts aus dem Repertoire der gewöhnlichen Umgangssprache bediene oder zumindest nicht allzu stark von der gebräuchlichen Redeweise abweiche (Aristot. rhet. III 2, 20, 1404b). 


\subsubsection{Plötzlicher Abbruch, unerwarteter Wechsel und unverbundene Rede als Verstärkungsmittel}

Neben der Rohbelassenheit der Sprache können in der antiken Rhetorik auch gröbere Risse im Redefluss legitimiert werden, welche der Forderung nach einem harmonischen Gepräge noch deutlicher zuwiderlaufen. Grundsätzlich sollte der Redefluss laut Quintilian immer wieder durch kurze Denk- und Ruhepausen unterbrochen werden (Quint. inst. IX 4, 56): «Erstens weil jeder Gedanke sein Ende hat und eine natürliche Pause verlangt [...]; sodann, weil die Ohren, die der fortlaufenden Rede folgen und sich gleichsam von dem Stromgefälle des Redeablaufs führen lassen, dann aufmerksamer urteilen, wenn das Ungestüm zum Halten kommt und Zeit zum Betrachten gibt» (Quint. inst. IX 4, 61). Keinesfalls jedoch dürften diese Pausen hart (durus) oder abgerissen (abruptus) erklingen (Quint. inst. IX 4, 62), ja generell sollte die «abgerissene [abruptus] Art zu reden» vermieden werden (Quint. inst. IV 2, 45; auch XII 10, 80). In ähnlicher Weise ermahnt Cicero die Redner, darauf zu achten, dass ihre Rede nicht zerfalle, regellos abschweife oder unvermittelt abbreche (Cic. de orat. III 190).

Trotz aller Ermahnungen zu einem harmonisch fortlaufenden, dezent rhythmisierten Redefluss benennt die klassische Rhetorik auch Einzelmomente, in denen Redner ihren Redefluss ganz bewusst abreißen oder versiegen lassen dürfen. Die sogenannte Aposiopese (auch: Reticentia, Cic. de orat. III 205 oder Praecisio, Rhet. Her. IV 30, 41 bzw. Abscisio, Quint. inst. IX 2, 54, 67) bezeichnet eine abrupte und plötzliche Unterbrechung der Rede, das Verstummen mitten im Satz, ein Verschweigen oder Ungesagt-Lassen von Worten (Quint. inst. IX 2, 54; 3, 60). Der Auctor ad Herennium definiert die Praecisio als ein «Abbrechen eines Gedankens», das dann vorliege, «wenn nach einigen Worten der übrige Gedankengang, den man auszusprechen begonnen hat, unvollendet bleibt» (relinquitur inchoatum) (Rhet. Her. IV 30, 41). Die Praecisio bzw. Aposiopese lässt sich in zweifacher Weise als Imperfektion verstehen: Sie stört nicht nur die Harmonie des Redeflusses, sondern sie drückt sich auch in einer «nicht zum Ende geführten Redeform» (inperfectus sermo) aus (Quint. inst. IX 2, 57), in der ein Gedanke unvollendet belassen wird (Rhet. Her. IV 30, 41). Gerade dieses Unvollendete kann vom Redner gewollt und punktuell äußerst effektvoll eingesetzt werden. Denn einerseits lassen sich, so Quintilian, durch den abrupten Abbruch der Rede Zorn und andere starke Affekte zur Schau stellen, wie etwa im bekannten Drohruf «Quos ego - » («Euch werde ich - !») aus Vergils 〈Aeneis〉 (Quint. inst. IX 2, 57). Andererseits könne der Redner damit bewusst etwas verschweigen (tacere), was eigentlich der Ausführung bedürfe (Quint. inst. IX 3, 60-61). Der Auctor ad Herennium hebt ebenfalls die besondere Kraft hervor, die sich aus einer unausgesprochen gebliebenen Vermutung entwickeln kann: Durch das plötzliche Verstummen 
gewinne das Ungesagte an Gewicht, was die Rede noch «unbarmherziger» wirken lasse als eine «beredte Darstellung» (Rhet. Her. IV 30, 41). Der bewusste Verstoß gegen grammatikalische und inhaltliche Vollständigkeit dient hier also der Verstärkung und Emotionalisierung der (unausgesprochen gebliebenen) Aussage.

Eine Nebenform der Aposiopese ist die «gleichsam vor dem rechtmäßigen Ende abgerissene Redeform» (Quint. inst. IX 2, 57). Obschon nur spärlich beschrieben, kann dieses Stilmittel anhand der genannten Beispiele so interpretiert werden, dass der Redner seine Rede zwar zum Abschluss bringt, einen bereits angefangenen Gedanken jedoch in unerwarteter Weise fortsetzt (Quint. inst. IX 2, 57). Bereits bei Demetrios wird der inkonsequenten Formulierung, d.h. einem «Mangel an Zusammenhang» des Gesagten mit dem Vorangehenden, ein gewisser Reiz attestiert, da dies für das Publikum unerwartet komme und rätselhaft erscheine (Demetr. eloc. III 153). Ein Aneinanderreihen nicht zueinander passender Redeteile oder ein plötzliches Umdisponieren mitten im Redefluss wird auch als Anakoluth bezeichnet. Das Anakoluth steht wegen seinem «inkonsequenten Satzbau» bzw. dem «Konstruktionswechsel» innerhalb der Äußerung im Gegensatz zur Akoluthie, also der korrekten Abfolge und Anordnung der Wörter im Satz (vgl. Sanders 1992: Sp. 485-487). Eine Rede, in welcher ein Teil so gesetzt werde, dass er sich relativ zum Vorhergehenden oder Folgenden nicht passend einfüge, ist denn auch Quintilian zufolge grundsätzlich als Solözismus, d.h. Syntaxfehler, zu bewerten (Quint. inst. I 5, 51). Solche Brüche in der Satzkonstruktion können als Anzeichen der Nachlässigkeit oder Unachtsamkeit des Redners gedeutet werden, gelten jedoch als häufiges Merkmal der mündlichen Rede, das vor allem in der unvorbereiteten Rede oder in Alltagsgesprächen oft vorkommt (vgl. Sanders 1992: Sp. 487). Aus diesem Grund lässt sich das Anakoluth aber auch als wirksames Redemittel einsetzen: Es verleiht der Rede einen authentischen Anstrich und eine besondere Lebhaftigkeit. Es kann von entspannter Lockerheit oder Nonchalance zeugen oder umgekehrt eine große emotionale Erregung des Redners anzeigen.

Ein abgerissener Redefluss kann schließlich auch durch die Unterdrückung von Verbindungsworten bzw. die unverbundene Aneinanderreihung von Wörtern erzeugt werden. Das Asyndeton (Unverbundene) ist innerhalb einer Rede grundsätzlich zu missbilligen, worauf schon Aristoteles hinweist (Aristot. rhet. III 12, 4, 1413b). Allerdings lasse sich die unverbundene Wortfolge auch dazu verwenden, Abwechslung in den gleichförmigen Tonfall der Rede $\mathrm{zu}$ bringen oder eine Aussage zu steigern und hervorzuheben. So wirke der unverbundene Ausspruch «Ich kam, sprach, bat» intensiver, als wenn Konjunktionen die drei Verben miteinander verbänden (Aristot. rhet. III 12, 4, 1413b). Bei Cicero findet die unverbundene Anreihung als Ausdrucksfigur des Dissolutum eigens Erwähnung (Cic. de orat. III 207). Diese mache sich dadurch bemerkbar, dass «die Verbindungsworte 
unterdrückt und so mehrere Worte unverbunden ausgesprochen werden» (Cic. orat. 39-41, 134-139, zit. in Quint. inst. IX 1, 39). Quintilian sieht die Funktion der Dissolutio darin, die Eindringlichkeit der Rede zu verstärken: «Die Wörter werden so einzeln eingehämmert und wirken dadurch so, als wären es noch mehr» (Quint. inst. IX 3, 50). Neben diesem 〈Einhämmerungseffekt〉 könne mit der unverbundenen Rede auch Kürze erreicht werden (brachylogía, brevitas), um einer Rede Deutlichkeit und Dringlichkeit zu verleihen. Gemäß Aristoteles eignet sich das Asyndeton besonders für den Redeabschluss, um den Schlusspunkt zu markieren: «Ich habe gesprochen, ihr habt es gehört, ihr wißt Bescheid, entscheidet!» (Aristot. rhet. III 19, 6, 1420a). ${ }^{1}$

\subsubsection{Abschweifung: Überflüssige Worte zur Umlenkung der Aufmerksamkeit}

Wie das Unverbundene oder Abgerissene muss auch alles, was nichts zur ausgeführten Sache beiträgt, aus Sicht der klassischen Rhetorik als Imperfektion betrachtet werden und ist als Fehler des Überflüssigen (perissología) im Normalfall abzulehnen. So sollten Umschreibungen oder unklare Ausdrücke (Amphibolien) laut Aristoteles sowohl im Dienste des guten Stils als auch des korrekten Ausdrucks vermieden werden (Aristot. rhet. III 5, 3-4, 1407a-b). Das Abschweifen ebenso wie das Herumreden um eine Sache innerhalb einer Rede ist als tadelnswertes Verhalten zu betrachten. Cicero rät denn auch dazu, den Redegegenstand gleich zu Beginn zu definieren, damit die Rede nicht aufgrund unterschiedlicher Verständnisse «zwangsläufig abschweift [vagari] oder in die Irre geht» (Cic. de orat. I 209). Aristoteles wiederum versucht das Abschweifen vom Sachverhalt aus einem anderen Grund zu unterbinden: Die Abschweifung diene dem Redner lediglich dazu, den Richter zu verwirren, um ihn zu Zorn, Neid oder Mitleid zu reizen - was für Aristoteles jedoch nicht die Aufgabe des Redners ist. Vielmehr solle jeder Redner auf die Aufklärung der tatsächlichen oder zumindest der wahrscheinlichsten Begebenheit hinwirken (Aristot. rhet. I 1, 4-8, 1354a-b). Gleichwohl hält Aristoteles das Einarbeiten unverständlicher Ausdrücke oder umständlicher Umschreibungen in eine Rede für wirkungsvoll:

Das machen Leute, wenn sie nichts zu sagen haben, aber vorgeben, etwas von Bedeutung zu sagen [...]. Das wortreiche Umkreisen des Eigentlichen lenkt ja nur ab, und den Zuhörern geht es dabei wie der Menge mit den Wahrsagern. Wenn diese sich in Unklarheiten ergehen, nicken sie ihnen beifällig zu. (Aristot. rhet. III 5, 4, 1407a)

1 Mit diesem Asyndeton setzt Aristoteles denn auch geistreich das Schlusswort seiner Abhandlung zur «Rhetorik». 
Trotz seiner Abneigung gegen solcherlei Aufbauschungs- und Ablenkungsmanöver schließt Aristoteles sie als Strategie nicht kategorisch aus. Müsse nämlich jemand eine schlechte Sache vertreten, so sei es besser, «bei jedem anderen Thema zu verweilen, nur nicht beim Gegenstand der Rede. Daher beantworten Sklaven Fragen nicht sofort, sondern halten uns mit Vorreden hin» (Aristot. rhet. III 14, 10, 1415b). Auch Cicero hält es manchmal für nützlich, «von seinem Thema, das man sich gestellt hat und behandelt, abzuweichen [digredi], um die Gemüter zu erregen» (Cic. de orat. II 311). Die ausschweifende Rede kann effektvoll zu einem wichtigen Punkt überleiten: «Am wirkungsvollsten [ornatissimus] also sind die Reden, die besonders weit ausgreifen [vagari] und von der individuellen, einzelnen Streitfrage $\mathrm{zu}$ einer wesentlichen und grundsätzlichen Erklärung führen» (Cic. de orat. III 120). In Ciceros bekannter Figurenliste findet die Abschweifung gleich unter verschiedenen Bezeichnungen Erwähnung: Als Gedankenfiguren genannt werden die Digressio, welche nicht beliebig in eine Rede eingefügt werden solle, sondern «dann, wenn man sich an ihr erfreut hat, wieder geschickt und elegant zur Sache führen muß» und die Declinatio brevis a propositio, was mit «beiläufige Bemerkung» übersetzt wird (Cic. de orat. III 203); bei den Ausdrucksfiguren die Digressio als Exkurs sowie eine weitere Form der Declinatio (eigentlich: 〈Abbiegen〉) (Cic. de orat. III 207). Diese Vielfalt abschweifender Stilmittel lässt vermuten, dass sich in der Antike so mancher Redner die Unsitte des Abschweifens zur Tugend gemacht hat.

Mit der Abschweifung verwandt ist das - inhaltlich unnötige oder zumindest unpräzise - Herumreden um eine Sache. Auch diese Redeform ist aufgrund ihrer Überflüssigkeit und Ungenauigkeit grundsätzlich zu verwerfen, kann aber wiederum in der Figur der Períphrasis oder Circumscriptio als Schmuckmittel verwendet werden (Quint. inst. VIII 6, 59-61; Cic. de orat. III 207). Manchmal könne ein «Drumherumreden` sogar zwingend werden, wie Quintilian behauptet, «wenn es nämlich verhüllt, was häßlich zu sagen ist», etwa indem Sallust sage, es sei zu dem gekommen, «was die Natur verlangt» (Quint. inst. VIII 6, 59). Hier wird aus der Not, das Hässliche zu vermeiden, eine Tugend gemacht, indem die gemeinte Sache nicht direkt benannt, sondern kunstvoll ausgelassen bzw. umschrieben wird.

\subsubsection{Patina- oder Neuheitseffekt durch Abweichungen von der aktuell gültigen Redeweise}

Eine spezielle Form der Imperfektion, die aus einer bewussten Abweichung vom Sprachgebrauch resultiert, sind die altertümliche Redeweise bzw. Vetustas und der Neologismus. Beide Formen weichen von der Art und Weise ab, wie im Hier 
und Heute geredet wird, indem sie entweder früher gebräuchliche oder noch nicht verbreitete Redeweisen verwenden. Mit Quintilian lässt sich Vetustas als Strategie begreifen, bei der absichtlich altertümliche Wörter in eine Rede eingewoben werden, um diese weihe- oder würdevoll, attraktiv, bewundernswert oder auch vertrauenerweckend erscheinen zu lassen (Quint. inst. VIII 3, 27; I 6, 39). Dieser kunstvoll bis künstlich hergestellte Anschein von «Alterspatina», welche laut Quintilian auch «bei den Bildwerken so hoch geschätzt wird» (Quint. inst. VIII 3, 25), soll einer Rede das «gewisse Etwas〉 verleihen. Aufgrund ihres Alters würden gewisse Wörter nämlich «einen noch angenehmeren Glanz» entfalten (Quint. inst. VIII 3, 27). Den Effekt von Altehrwürdigkeit (antiquitas, priscum) versuchten laut Cicero einige Redner auch durch eine bäurisch raue, ungehobelte Stimme hervorzurufen (Cic. de orat. III 42). Bei Cicero wird diese Taktik allerdings als wenig authentisch entlarvt, da die «echte` altertümliche Art zu reden keineswegs rau, unkultiviert oder abgehackt sei, sondern vielmehr ebenmäßig und flüssig. Man würde damit nicht die altehrwürdigen Redner, sondern lediglich die Erntehelfer nachahmen (Cic. de orat. III 45).

Mit diesem Einwand dementiert Cicero aber nicht die grundsätzlich positive Wirkung von Alterspatina. Denn es wirkten die schönen und vielfältigen Farben auf neuen Gemälden zwar viel frischer als auf alten: «Doch wenn sie uns beim ersten Anblick auch für sich einnehmen, gefallen sie uns doch nicht länger; dagegen fesselt uns an altertümlichen (antiquis) Gemälden gerade das Schmucklose, Unscheinbare» (Cic. de orat. III 98). Die Dezenz alter Gemälde wird hier in Kontrast gesetzt zum intensiven, aber vergänglichen Reiz des Neuen. Was für die Bilder und ihre Farben gesagt wird, gilt offenbar auch für gesprochene Worte. Obschon uns für gewöhnlich das Neue, Makellose und Unbenutzte attraktiv erscheint, während wir das Alte für abgenutzt und weniger perfekt halten und obschon eine der wesentlichen Elaborationsweisen der klassischen Rhetorik darauf abzielt, die Rede in Glanz erstrahlen zu lassen, können gerade auch Anzeichen von Alter und Abnutzung eine positive Wirkung entfalten. Außerdem bemerkt Quintilian, dass alte Wörter, wenn ihr Gebrauch geruht habe, plötzlich wieder «wie neu» wirken könnten (Quint. inst. I 6, 39). Wird der Effekt der Alterspatina bewusst erzeugt, gelte es (wie immer) Maß zu halten und keine allzu veralteten Ausdrücke zu verwenden, denn die Gefahr der «Altertümelei» lauere jederzeit (Quint. inst. VIII 3, 25, 30; I 6, 40).

Anders als veraltete Ausdrücke verstoßen Neologismen per definitionem gegen die Sprachrichtigkeit, da sie weder in der aktuell geltenden noch in einer früheren Sprachlehre vorgesehen sind. Bereits die klassische Rhetorik ist sich jedoch im Klaren darüber, dass Sprache sich immer wieder wandelt und Neuschöpfungen eine wichtige Quelle der Sprachkunst sind: «Denn auch, was jetzt alt ist, war einmal neu, und manche [Wörter] sind im Gebrauch, die recht junge 
Bildungen sind» (Quint. inst. VIII 3, 34). Neologismen dürfen daher in einer Rede vorkommen. Zumindest bei gewagten Neubildungen sollten sich Redner, wenn es nach Quintilian geht, aber mittels einer Abschwächung absichern, beispielsweise mit Wendungen wie: «[ $[\mathrm{u}] \mathrm{m}$ es so auszudrücken; wenn der Ausdruck gestattet ist; gewissermaßen; gestattet mir diesen Ausdruck zu gebrauchen»» (Quint inst. VIII 3, 37). Das explizite Abschwächen der eigenen Gewandtheit oder Sicherheit im Sprechen durch den Redner selbst kann als separate Strategie der Imperfektion in der Rede eingesetzt werden, wie wir im kommenden Abschnitt genauer sehen werden.

\subsection{Das Ethos der Bescheidenheit: Vom gezielten Herabsetzen der Kunst}

Um mit ihrer Rede Anklang zu finden und zu überzeugen, müssen Redner gemäß der klassischen Rhetorik das Wohlwollen des Publikums gewinnen und dieses versöhnlich stimmen (captatio benevolentiae, conciliare). Persuasion findet nicht nur auf der Ebene des Inhalts, der Argumentation und der gewählten Ausdrucksweise statt, sondern hängt wesentlich vom Eindruck ab, den das Publikum vom Redner selbst und von seiner Persönlichkeit erhält. Auf der Wirkungsebene des Ethos durchdringt der Charakter des Redners die gesamte Rede. Zwischen dem Ethos des Redners und der Aufgabe des Versöhnlich-Stimmens besteht eine enge Verbindung: die Gunst des Publikums hängt davon ab, wie es den Redner wahrnimmt, und diese Wahrnehmung wiederum wird davon beeinflusst, ob es dem Redner wohlgesinnt ist. Deshalb versucht die Rhetorik seit der Antike auch Anleitung dazu zu geben, wie Redner das Bild beeinflussen können, das sie von sich selbst abgeben. Dieses Unterfangen gestaltet sich jedoch komplex: Cicero macht darauf aufmerksam, dass die drei Möglichkeiten des Überzeugens - docere, conciliare, permovere - in unterschiedlicher Weise umgesetzt werden müssen: Während die Informationsabsicht (docere) offen zur Schau getragen werden solle, müssten die beiden anderen Ziele, insbesondere das Gewinnen der Gunst (conciliare), aber auch die Beeinflussung der Gefühle (permovere), im Verborgenen bleiben, so dass es «den Anschein hat, als wollten wir nur informieren; die beiden anderen müssen wie Blut im Organismus im Zusammenhang der Rede fließen» (Cic. de orat. II 310). Jeder Redner solle, ermahnt Aristoteles, darum bemüht sein, sich vor dem Publikum in ein positives Licht zu rücken. Doch dürfe er sich bei diesem Versuch keinesfalls ertappen lassen: «[S]telle auch dich selbst sofort als einen Mann von einem bestimmten Charakter dar, damit die Hörer dich als solchen betrachten, und ebenso deinen Gegenspieler, gehe dabei aber so vor, daß man es nicht merkt!» (Aristot. rhet. III 16, 10, 1417b). 
Dass man beim Reden generell eine unauffällige Natürlichkeit ausstrahlen sollte, um den Zuhörern nicht verdächtig zu werden, haben wir weiter oben schon gesehen. So soll auch das Einholen von Sympathien nicht allzu offensichtlich betrieben werden. Laut Quintilian dürfe etwa in einer witzig vorgetragenen Passage keinesfalls der Eindruck erweckt werden, «nach Lacheffekt zu haschen», denn es gebe «nichts Abgeschmackteres als das, was schon so vorgetragen wird, als wäre es voll witzigen Salzes» (Quint. inst. VI 3, 26). Wie der Redner sein Publikum für sich gewinnen kann, ohne diesem seine Absicht allzu offensichtlich unter die Nase zu reiben, und welche Rolle dabei die Imperfektion spielt, soll im Folgenden genauer untersucht werden.

\subsubsection{Bescheidenheit und Redlichkeit: Ethos-Wirkungen des Understatement}

Glaubwürdigkeit ist ein wertvolles Gut des Ethos, das es zu pflegen und zu wahren gilt, um ein Publikum überzeugen zu können: «Durch den Charakter [ếthos] geschieht dies, wenn die Rede so dargeboten wird, daß sie den Redner glaubwürdig erscheinen lässt. Den Anständigen glauben wir nämlich eher und schneller» (Aristot. rhet. I 2, 4, 1356a). Aristoteles stellt hier einen Zusammenhang zwischen dem Charakter, der Glaubwürdigkeit und der Überzeugungskraft des Redners her. Nur ein Redner, der als Person integer wirkt, kann das nötige Vertrauen des Publikums erlangen, um es von seinen Ansichten zu überzeugen. Auch laut Quintilian kann nur ein Redner das Wohlwollen und die Sympathien des Publikums gewinnen, der dem Publikum liebenswürdig, freundlich, menschlich, rechtschaffen und natürlich erscheint (Quint. inst VI 2, 13-19). Ebenso wichtig wie eine freundliche Erscheinung kann das Bezeugen einer gewissen Zurückhaltung und Bescheidenheit sein, wie sich einer Stelle aus Ciceros «De Oratore» entnehmen lässt:

Anzeichen von Umgänglichkeit und von Großzügigkeit, von Sanftmut und Anhänglichkeit, von Dankbarkeit und Bescheidenheit zu zeigen, ist sehr nützlich. Alles, was für rechtschaffene und schlichte Charaktere ohne Ungestüm, Hartnäckigkeit, Streitsucht und Härte typisch ist, gewinnt die Sympathie in hohem Maße und entzieht sie denen, bei denen diese Eigenschaften nicht vorhanden sind. (Cic. de orat. II 182)

Auch wenn Überzeugungskraft gemeinhin mit Selbstsicherheit und einem perfekten Auftritt assoziiert wird, kann es für den Redner gerade im Sinne seiner Glaubwürdigkeit und Integrität angebracht sein, nicht allzu selbstbewusst aufzutreten, da ihm dies als Zeichen der Arroganz oder Überheblichkeit angekreidet werden könnte. So deutet Quintilian «alles Großtun mit der eigenen Person» und ganz besonders das «Prahlen mit seiner Beredsamkeit» als Fehler der Anmaßung, 
der beim Publikum Widerwillen oder sogar Hass provozieren könne (Quint. inst. XI, 1, 15). Dies gelte insbesondere, wenn man sich falsche Geltung anmaße, aber auch dann, wenn echte Verdienste oder überragende Kenntnisse vorzuweisen seien. Selbst wenn die eigenen Fähigkeiten hervorragend seien, solle man sich, wie Quintilian meint, lieber bescheiden geben als überheblich zu wirken (Quint. inst. XI 1, 17).

Bei Cicero wird dieser Punkt an einer Stelle verschärft, wo der große Redner Crassus eingesteht, immer wieder die Erfahrung zu machen, dass er «bei den ersten Worten einer Rede vor Angst erbleiche und von ganzem Herzen und an allen Gliedern bebe» (Cic. de orat. I 121). Diese für einen routinierten Redner erstaunliche Schamhaftigkeit (mirificus pudor) scheint jedoch seiner Rede nicht zu schaden, sondern vielmehr einen Nutzen zu bringen, «da sie seine Redlichkeit [probitas] empfahl» (Cic. de orat. I 122). Die Einsicht, dass Schüchternheit die Rechtschaffenheit des Redners erweisen kann, mag für all jene, die vor einer Rede nervös oder verunsichert sind, ein gewisser Trost sein - und für die allzu Selbstsicheren eine Mahnung. Crassus sieht es als eine große Aufgabe und Last, vor einer größeren Menge und in einer wichtigen Angelegenheit zu reden (Cic. de orat. I 116). Aus diesem Grund sei es angebracht, mit Zurückhaltung oder gar Beschämung aufzutreten - was auch für jene gelte, die gekonnt und mit Leichtigkeit zu reden verstünden. In seinen Augen wäre es unverschämt, wenn Redner angesichts ihrer wichtigen Aufgabe «nicht scheu das Wort ergreifen und am Beginn der Rede Zeichen von Verwirrung zeigen» würden (Cic. de orat. I 119). Etwas «Lampenfieber ist also durchaus schicklich.

\subsubsection{Dissimulatio Artis: Das kunstvolle Verbergen der Kunst}

Die antike Rhetorik offenbart eine sensible Wahrnehmung für die Gefahren, die in der Zurschaustellung der eigenen Kunst liegen. Bereits der bloße Anschein von Kunstfertigkeit könnte schädlich sein für die Glaubwürdigkeit des Redners. Denn beim Publikum darf auf keinen Fall der Eindruck erweckt werden, dass der Redner trickst. Damit die Rede nicht als einstudiertes Spektakel durchschaubar wird, das gezielt auf das Publikum einwirken will, muss die darin angewendete Kunst kunstvoll verborgen werden. Die Idee der Verbergung der Kunst bzw. Dissimulatio Artis wird in den antiken Künsten breit angewendet, um den Kunstgenuss zu steigern - doch in keiner Kunst wirkt sich das Bewusstsein über die Künstlichkeit und Inszeniertheit zugleich so schwerwiegend auf das Ansehen des Absen- 
ders aus wie in der Rhetorik. Als Erster ${ }^{2}$ weist Aristoteles auf die Notwendigkeit hin, in der Rede die Kunst zu verbergen, da bei einem offensichtlichen Bemühen oder einem allzu kunstvollen Arrangement die Zuhörer argwöhnen könnten, dass der Redner etwas heimlich gegen sie im Schilde führe - «ähnlich wie wenn Wein gepanscht wird» (Aristot. rhet. III 2, 4, 1404b).

Aus diesem Grund rät Aristoteles gleichsam zu «Meta〉-Kunstgriffen, welche dabei helfen sollen, die Elaboriertheit der eigenen Rede im Verborgenen zu halten. Ein Trick, seine Redekunst gut zu verbergen (eú kléptetai), wäre es laut Aristoteles, die Rede so zu komponieren, dass man «seinen Wortschatz aus der gewöhnlichen Umgangssprache nimmt» (Aristot. rhet. III 2, 5, 1404b). Die Nähe zur unelaborierten Alltagssprache soll dabei den Eindruck der Künstlichkeit verhindern. Ein anderer Kniff wäre es, die Elemente der Rede bewusst nicht allzu stimmig zu arrangieren: Zwar wäre es unglaubwürdig und dem Prépon bzw. Angemessenen zuwiderlaufend, etwas «Hartes» in weichem Ton vorzutragen, doch dürfe die Vortragsweise auch nicht zu offensichtlich mit dem Inhalt in Abstimmung gebracht werden. Würden bewusst hart klingende Wörter auch noch durch die Stimme und Mimik des Vortrags unterstrichen, könnten sich die Zuhörenden hinters Licht geführt fühlen. Damit die mit der Rede evozierte Härte nicht inszeniert wirke, sei auf diese zusätzliche Betonung zu verzichten (Aristot. rhet. III 7, 10, 1408b). Eine bewusste Reduktion des Elaborationsgrads soll auch hier zu vermeiden helfen, dass sich die Künstlichkeit und Absicht der Rede zeigt.

Die lateinischen Rhetoriker haben sich in analoger Weise der Regel verschrieben, die eigene Kunst nicht zur Schau zu stellen. Denn dort, wo die Kunst im Vordergrund stehe, scheine man es mit der Wahrheit nicht allzu genau zu nehmen: «ubicumque ars ostentatur, veritas abesse videatur» (Quint. inst. IX 3, 102). Was das Minen- und Gebärdenspiel des Redners beim Vortrag betrifft, solle eine gewisse Natürlichkeit bewahrt werden. Zwar könnten laut Quintilian Redner hierin einiges von der Schauspielkunst lernen, doch dürfe damit keinesfalls übertrieben werden, «denn wenn es hierfür bei den Redenden einer Kunst bedarf, so vor allem der, nicht als Kunst zu erscheinen» (ne ars esse videatur) (Quint. inst. I 11, 3). Der Auctor ad Herennium betont, eine allzu offensichtliche Elaboration der Rede würde die Wahrscheinlichkeit der Aussage mildern und somit der Wahrhaftigkeit des Redners schaden (Rhet. Her. IV 22, 32). Besonders kunstvolle Weisen der Ausschmückung seien deshalb nur mit äußerster Sparsamkeit zu verwenden, da sie den Eindruck erweckten, nicht ohne Ausarbeitungsaufwand und Mühe (sine elaboratione et sumptione operae) aufgefunden werden zu können (Rhet. Her. IV 22, 32). Wenn ein Stilmittel wie die Aufzäh-

2 Laut dem «Historischen Wörterbuch der Rhetorik» finden sich in der «Rhetorik〉 des Aristoteles die «ältesten systematischen Überlegungen» zur Verbergung der Kunst (Till 2009: Sp. 1038). 
lung allzu ausgefeilt angewendet werde, könne dies beim Zuhörer den «Verdacht eines geschickt gewählten Kunstgriffes» erwecken: «das aber nimmt der Rede ihre Glaubwürdigkeit» (Rhet. Her. I 10, 17). Eine zu ausführliche oder allzu weit in die Rede zurückführende Aufzählung scheine dem Publikum zeigen zu wollen, unter welch großen Mühen sie vorbereitet wurde und stelle damit die Kunstfertigkeit und den Geistreichtum des Redners heraus. Gerade das sei aber tunlichst zu vermeiden (Rhet. Her. II 20, 47).

Den Zusammenhang zwischen dem Sichtbarwerden der Kunst und dem Verlust der Glaubwürdigkeit erkennt auch Cicero: Sowohl Glanz (splendor), Anmut (festivitas) als auch eine kunstvolle Anordnung (conncinnitas) - also Merkmale höchster Elaboration - seien in der Einleitung zu meiden, weil sie den Verdacht erweckten, der Redner gehe kalkuliert und künstlich vor, was der Rede ihre Glaubwürdigkeit und dem Redner seine Autorität nehme (Cic. inv. I 25). Auch Maßnahmen zur Rhythmisierung der Rede sollten besser verhüllt werden: Cicero rät dazu, innerhalb einer Rede häufig den Rhythmus zu wechseln, um den Eindruck abzuwenden, «das, was wir tun, planmäßig und bewußt zu tun» (Cic. de orat. III 193). Quintilian empfiehlt einen möglichst natürlich erscheinenden Redefluss: «Das Verbergen der Sorgfalt [dissimulatio curae] ist besonders wichtig, damit es so aussieht, als habe sich der Rhythmus von selbst eingestellt [ut sponte fluxisse] und sei nicht herbeigeholt und erzwungen worden» (Quint. inst. IX 4, 147).

Gemäß der antiken Auffassung wirft es kein gutes Licht auf den Redner, wenn eine Rede kunstvoll bearbeitet zu sein scheint - auch wenn sie durchaus nach den Regeln der Kunst elaboriert sein sollte. Der Auctor ad Herennium hält es zum Erlernen der Redekunst für nötig, sich mit auffällig kunstvollen Beispielen zu befassen, für die Praxis müsse dann aber gelernt werden, die Kunst gut versteckt halten: «[S]päter bei der Rede wird die Lehre durch die Fähigkeit des Redners verborgen, damit sie nicht herausstechen und von allen gesehen werden kann» (Rhet. Her. IV 7, 10). Ist die Redekunst vom Redner erst einmal verinnerlicht, solle er darauf achten, dass seine Kunst beim Sprechen nicht mehr «durchscheinen» wird (ne ars appareat) (Rhet. Her. IV 7, 10). Wir haben es also mit einer ambivalenten Haltung, ja fast schon mit einem Widerspruch zu tun: Mit der Forderung nach Elaboriertheit, nach Vollendung der Kunst, die sich jedoch nicht zeigen darf. Also mit einer übergeordneten Kunst, bei der die wahre Kunst des Redners darin besteht, die eigene Kunst zu verbergen: 〈Ars est celare artem〉. 


\subsubsection{Stegreifrede: Schlagfertigkeit, Spontaneität und sympathische Nachlässigkeit}

Ein Verbergen der kunstvollen Ausarbeitung kann auch dazu dienen, der Rede einen Anstrich von Spontaneität zu verleihen. Laut Auctor ad Herennium sei gleich zu Beginn der Rede darauf zu achten, «dass die Rede nicht eigens vorbereitet erscheint» (ut non adparata oratio videatur esse) (Rhet. Her. I 7, 11). Eine spontane Wirkung soll selbst dann suggeriert werden, wenn die Rede zuvor sorgfältig ausgearbeitet wurde. Kommt die Rede unvorbereitet daher, wird der Eindruck vermieden, dass der Redner etwas im Schilde führen könnte. Die Rede gewinnt an Leichtigkeit und gegebenenfalls auch an Vehemenz. Es erstaunt denn auch nicht, dass die Fähigkeit zum unvorbereiteten Sprechen, zur sogenannten Stegreifrede (subito dicere, Cic. de or. I 150; ex tempore dicere, Quint. inst. X, 7, 4) in der antiken Rhetorik einen hohen Stellenwert besitzt. Das Reden aus dem Stegreif hat ihr großes Vorbild in der Person des Sokrates bei Platon, der selbst der Verschriftlichung von Gedanken noch skeptisch gegenübersteht (siehe z.B. Plat. Phaidr.). Vermutlich entwickelte sich die Rhetorik als Kunstlehre, die sich mit der minutiösen Vorbereitung und Ausarbeitung der Rede beschäftigt, zunächst aus der spontanen und wenig vorstrukturierten Rede (vgl. Geißner 2007: 1359). Im Gegensatz zu einer am Schreibtisch entwickelten und wortwörtlich auswendig gelernten Rede ist es in einer unvorbereiteten Rede möglich, sich dem jeweiligen Moment (Kairós) anzupassen und dynamisch auf Fragen oder Einwände des Publikums zu reagieren. Der Stegreifrede haftet denn auch etwas «Spontanes, Ungestümes, oft Leidenschaftliches» an (Geißner 2007: 1359).

Etwas von der Schlagfertigkeit, von der Spontaneität und dem Ungestüm der Stegreifrede sollte sich nun mit Vorteil auch in der vorbereitete Rede erhalten. Nicht von ungefähr preist Quintilian an Cicero neben seiner herausragenden Eloquenz, seinem Talent, seiner Genauigkeit, seinem leidenschaftlichen Schwung, seinem Charme und seiner Autorität auch die besondere Fähigkeit, die eigene Redekunst zugleich komplett mühelos, spontan und selbstverständlich erscheinen zu lassen:

[D]abei strömt die Fülle alles dessen, wovon jemand kaum einen Teil mit angespanntester Mühe zuwege bringen könnte, ohne jede Spur von Anstrengung [fluunt inlaborata], und die Kunst einer solchen Rede, dergleichen man in solcher Schönheit nie gehört, enthüllt sich bei alledem wie leicht hingeworfen in einem glücklichen Augenblick [felicissimam facilitatem]. (Quint. inst. X 1, 111)

Der Anschein der Spontaneität und des Hingeworfenen bar jeder Bemühung kann eine intendierte Wirkungsweise selbst vorbereiteten Sprechens sein. Aber auch die wirklich unvorbereitete Rede will gelernt sein. Erst durch Fleiß und 
Übung könne ein Redner in der unvorbereiteten Form des Redens zuverlässig die gewünschte «Leichtigkeit und Geläufigkeit» (facilitates) in der Wirkung hervorbringen (Quint. inst. X 7, 8). Damit ein Redner für Situationen gewappnet sei, in denen er «sofort mit seiner Rede beginnen muß» (Quint. inst. X 7, 2), solle er sich immer wieder im Verschriftlichen von Reden üben, damit auch das, was scheinbar spontan hingeworfen (subito effusa) sei, durch die schriftliche Arbeit gefärbt werde. Zudem solle er eine große Zahl vorbildhafter Reden studieren, um sich die nötige Ausdrucksfülle anzueignen (Quint. inst. X 7, 2). Die Bereitschaft zur Stegreifrede könne sich ein Redner nur dann jederzeit erhalten, wenn er den öffentlichen Auftritt täglich übe - wenn möglich vor Personen, deren Meinung ihm wichtig sei (Quint. inst. X 7, 24). Routine wird somit als Voraussetzung für die Kunst der spontanen Rede angesehen. Zumindest der grobe Aufbau und der rote Faden sollten selbst bei spontanen Redeeinsätzen nach Möglichkeit vorher angedacht werden (Quint. inst. X 7, 5-6; 13). Falls nicht einmal hierzu Zeit bleibe, solle man sich mittels eines gemächlichen und von Ruhepausen durchzogenen Vortrags die nötige Zeit zum Überlegen schaffen, um sich nicht einem «nichtssagenden Worterguß» zu überlassen (Quint. inst. X 7, 22). Hinter der scheinbaren Leichtigkeit einer gelungenen Stegreifrede kann also sehr viel Arbeit und Überlegung stecken. Selbst in unvorbereiteten Situationen handelt es sich Quintilian zufolge denn auch nur um eine scheinbare Unüberlegtheit im Auftritt, «als gerade das, was ohne Überlegung vor sich geht, doch auf Überlegung beruht. Denn es handelt sich, scheint mir, gar nicht um Reden, falls jemand nicht in überlegter Anordnung mit bewußtem Wortschmuck und Wortschatz spricht, sondern um Wortgetümmel» (Quint. inst. X 7, 12). Die in der antiken Auffassung angestrebte Spontaneität ist also nicht das Resultat einer wirklich spontanen Handlung, sondern der Effekt einer «habitualisierten Regelbeherrschung», die nicht in die künstliche affectatio abgleitet» (Till 2009: Sp. 1038).

Cicero bewertet letztlich die Qualität einer Stegreifrede immer noch niedriger als jene einer sorgfältig elaborierten Rede: Die Stegreifrede sei als Übung durchaus zu empfehlen, doch könne eine spontan gehaltene Rede niemals an das «Werk reiflicher Überlegung und Besinnung» heranreichen (Cic. de orat. I 150). Dass ein Redner unvorbereitet spricht, kann jedoch unter Umständen auch nur vorgetäuscht sein: Als sehr erfolgreich stellt Cicero das gewiefte Vorgehen des Redners Antonius vor Gericht dar. Ohne jeden Anschein von Berechnung (nulla meditationis suspicio) und scheinbar gänzlich unvorbereitet (imparatus) beginne er jeweils seinen Vortrag, wobei er jedoch stets bestens vorbereitet (paratus) sei und von diesem Trick ließen sich die Richter Mal für Mal überrumpeln (Cic. Brut. 37, 139). Entsprechend hält es Quintilian in Gerichtsreden für eine Grundregel, «zu beachten, bestimmte Stellen gerade, die wir aufs beste rhythmisch gebunden haben, so vorzutragen, als wären sie ganz frei, und so auszusehen, als überlegten 
wir manchmal und wären im Zweifel, während wir das suchen, was wir schon ausgearbeitet mitgebracht haben» (et cogitantibus nonnumquam et dubitantibus similes quaerere videamur quae attulimus) (Quint. inst. XI 2, 47).

Damit die Rede nicht erstarrt und sich ihre Leichtigkeit bewahrt, sollten bei der Komposition die sprachlichen Regeln nicht allzu rigide angewendet werden. Ja, bei Fehlern, die nicht besonders schwer wiegen, jedoch nur mit Mühe ganz zu vermeiden wären, wird manchmal sogar zur Nachlässigkeit geraten. So sei es zwar zu vermeiden, dass die Vokale aufeinander folgender Wörter aufeinanderstoßen (was als Hiat bezeichnet wird), wodurch eine Rede auseinanderklaffen oder ins Stocken kommen könne. Gerade hier dürfe man sich aber, wie Quintilian meint, «nicht bange machen lassen wie vor einem entsetzlichen Verbrechen», sondern solle eine gewisse Sorglosigkeit oder Nachlässigkeit (neglegentia) walten lassen (Quint. inst. IX 4, 34-35). Cicero befindet sogar, der Hiat verrate die «nicht unsympathische Nachlässigkeit eines Mannes, dem es mehr um die Sache als um die Worte zu tun ist» (Cic. orat. 23, 77). Auch Nachlässigkeit kann zur Neglegentia diligens werden, also einem nur scheinbar nachlässigen und spontanen Gebaren, das in Tat und Wahrheit wohlberechnet als Wirkungsmittel eingesetzt wird (Cic. orat. 23, 78). Quintilian hält es für angebracht, «zuweilen bestimmte Stellen absichtlich gleichsam in der Wortfügung zu lockern, und zwar solche, die größte Mühe gemacht haben, damit man ihnen die Mühe nicht ansieht» (ne laborata videantur) (Quint. inst. IX 4, 144). Insgesamt fordert Cicero von Rednern eine Mischung zwischen einer «ruhigen Gelassenheit [lenitas], die uns den Zuhörern sympathisch macht» (conciliamur) und einer Leidenschaft (vis), «mit deren Hilfe wir dasselbe Publikum mitreißen» (excitamus) (Cic. de orat. II 212).

Selbst der Eindruck von Sorglosigkeit und Lockerheit lässt sich also künstlich erzeugen. Eine gewisse Nonchalance, Ungezwungenheit und Lässigkeit, welche die Kunst verbirgt, wurde in der frühen Neuzeit mit der Idee der Sprezzatura gar zum eigentlichen Ideal erhoben. ${ }^{3}$ Dieses Ideal deutet sich im antiken Begriff der Anmut (cháris, gratia), die wir ja als Elaborationsmerkmal der klassischen Rede kennengelernt haben, bereits an: Als «Reiz einer ungezwungenen Natürlichkeit» (Göttert 1992: 610) setzt Anmut den Anschein von Leichtigkeit voraus.

3 Der Begriff Sprezzatura oder Leichtigkeit entstammt Baldassare Castigliones «Libro del Cortegiano> (1528), in welchem dem Hofmann empfohlen wird, «[di] usar in ogni cosa una certa sprezzatura, che nasconda l'arte e dimostra ciò che si fa e dice venir fatto senza fatica e quasi senza pensarvi» (Castiglione 1986 [1528]: XXVI). 


\subsubsection{Selbsttadel: Herabsetzung der eigenen Fähigkeiten}

Um die Bescheidenheit, Redlichkeit und Glaubwürdigkeit herauszustellen und den Verdacht der Gerissenheit oder Berechnung von sich zu weisen, gibt die klassische Rhetorik dem Redner sogar Verfahren der expliziten Herabsetzung der eigenen Redekompetenz (dissimulatio oratoris) an die Hand. Bereits Aristoteles preist den Selbsttadel in der Rede als «Heilmittel gegen jede Übertreibung, das allbekannt ist: Der Redner soll nun sich selbst deswegen zurechtweisen! Das erweckt dann den Eindruck der Wahrheit, da dem Redner ja nicht entgeht, was er tut» (Aristot. rhet. III 7, 9, 1408b). Indem sich der Redner selbst tadelt, kann er dem Publikum Selbsterkenntnis, Transparenz und Aufrichtigkeit suggerieren. Quintilian zufolge kann sich vor Gericht ein Redner, der sich bewusst schwach, unvorbereitet und der Situation nicht gewachsen gebe, der Gegenseite stillschweigend empfehlen (commendatio tacita) und jeden Verdacht auf Ehrgeiz oder Böswilligkeit von sich weisen (Quint. inst. IV 1, 8). In Ciceros «Orator` wird als Wirkungsmittel sowohl die Selbstkorrektur empfohlen, wenn man sich als Redner selbst verbessere, als würde man sich tadeln (cum corrigimus nosmet ipsos quasi reprehendentes) (Cic. Orat. 39, 135), als auch der Selbstzweifel: Der Redner solle sich im Zweifel darüber geben, was er zu sagen oder worüber er eher $\mathrm{zu}$ sprechen habe (addubitet ecquid potius aut quo modo dicat) (Cic. orat. 40, 137). Ciceros Aufzählung der Gedanken- und Ausdrucksfiguren in «De Oratore` enthält eine ganze Gruppe von Stilmitteln, die auf Selbstbezichtigung und das Herabsetzen der eigenen Fertigkeiten des Redners abzielen: das ratlose Zweifeln (dubitatio), die Selbstberichtigung (correctio), das Zugeständnis (permissio) sowie den Selbsttadel bzw. die Richtigstellung (reprehensio) (Cic. de orat. III 201-208). ${ }^{4}$ Zur Dubitatio äußert sich Quintilian ausführlicher: Wenn wir unsere eigenen Worte anzweifelten, indem wir «so tun, als suchten wir, wo wir anfangen, wo aufhören sollten, was wir vor allem sagen, und ob wir überhaupt reden sollten», könnten wir einen «vertrauenswürdigen Eindruck von Wahrheit» vermitteln (Quint. inst. IX 2, 19).

Neben dem Suggerieren von Bescheidenheit, Wahrhaftigkeit und Arglosigkeit sieht der Auctor ad Herennium in der Correctio generell ein Stilmittel,

\footnotetext{
4 Die Correctio wird gleich zweimal aufgeführt, ein Mal unter den Gedankenfiguren als eine «Berichtigung, entweder ehe man oder nachdem man sich geäußert hat, oder indem man etwas von sich weist», das zweite Mal unter den Figuren des Ausdrucks als «eine andere Art der Richtigstellung» (alia correctio), wobei jedoch der angebliche Unterschied nicht weiter erläutert wird (Cic. de orat. III 203; 207). Analoges gilt für die Dubitatio, die zunächst als Figur des Gedankens aufgezählt wird, dann als Ausdrucksfigur: «noch einmal, doch in anderer Form, der Zweifel» (rursum alia dubitatio) - beide Male ohne weitere Erklärung (Cic. de orat. III 203; 207).
} 
welches «aufhebt, was gesagt wurde, und an seine Stelle das setzt, was geeigneter erscheint» (Rhet. Her. IV 26, 36). Dies könne auch dazu verwendet werden, den Zuhörer zu beeindrucken oder die Aufmerksamkeit auf einen Punkt zu lenken: «Denn der durch ein gewöhnliches Wort hervorgehobene Sachverhalt scheint nur ziemlich obenhin gesagt; nach der Berichtigung durch den Redner selbst wird er passender vorgetragen» (Rhet. Her. IV 26, 36). Anstatt gleich von Anfang an das beste Wort zu verwenden, könne man mittels einer Correctio auf die besondere Bedeutung eines Sachverhaltes oder Wortes aufmerksam machen (Rhet. Her. IV 26, 36). Man sagt also bewusst zunächst etwas Falsches oder wenig Passendes, um sich sofort zu korrigieren, so dass dadurch ein Kontrast entsteht, der das zuletzt Gesagte unterstreicht. Über eine explizite Herabsetzung der eigenen Fähigkeiten funktioniert auch die Communicatio, wie sie bei Quintilian erwähnt wird. Indem der Redner seinen Gegner oder den Richter um Rat frage, erhoffe er sich, den Betroffenen zu schmeicheln oder sie in Spannung zu halten (suspendire) (Quint. inst. IX 2, 20).

Ein weiterer Kunstgriff der Selbstkorrektur ist die in der «Rhetorica ad Herennium> beschriebene Occultatio. Sie liegt vor, «wenn wir sagen, wir übergingen oder wüßten nicht oder wollten nicht das sagen, was wir nun gerade erst recht sagen» (Rhet. Her. IV 27, 37). In der Occultatio wird ein performativer Widerspruch und damit ein eigentlicher Fehler begangen: Ich tue exakt das, wovon ich behaupte, ich würde es nicht tun. Der Nutzen der Figur liegt laut dem anonymen Autor darin, dass damit beispielsweise ein Verdacht «im Verborgenen erregt» werden könne, der aber anschließend vom Gegner nicht eigentlich zu widerlegen sei (Rhet. Her. IV 27, 37). Dieser Kunstgriff leitet bereits über zur Simulatio oder Verstellung, wie wir sie gleich im Detail kennenlernen werden. ${ }^{5}$

5 Eine besondere Form der Herabsetzung der eigenen (oder auch fremder) Fähigkeiten, die sich allerdings nicht auf die Redekunst selbst bezieht, ist die Deminutio. Sie liegt laut Auctor ad Herennium dann vor, «wenn wir sagen, wir oder diejenigen, die wir verteidigen, besäßen von Natur aus oder durch das Schicksal oder durch eigenen Fleiß eine herausragende Eigenschaft, die aber in der Rede abgeschwächt und vermindert wird, damit nicht eine anmaßende Prahlerei an den Tag gelegt wird» (Rhet. Her. IV 38, 50). Lieber also die eigenen Fähigkeiten - oder jene der Person, die der Redner in gutem Licht erscheinen lassen möchte - als weniger perfekt hinstellen, als sie sind, als sich dem Vorwurf von Anmaßung oder Prahlerei auszusetzen. Auch Cicero erwähnt, dass eine überragende oder glänzende Stellung Neid erregen könne, weshalb man dafür zu sorgen habe, dass dieser Eindruck abgeschwächt werde (Cic. de orat. II 210). 


\subsubsection{Simulatio: Verstellung, Ironie und Witz zur Einschmeichelung und Ablenkung}

Es gibt nun Fälle, in denen die Captatio benevolentiae nicht ohne Weiteres möglich ist und wo gemäß der klassischen Rhetorik die sogenannte Einschmeichelung (insinuatio) zum Zuge kommen sollte: Gewissermaßen «versteckt durch Verstellung» (occulte per dissimulationem) soll so bewirkt werden, dass der Redner zu einer günstigeren Ausgangslage für seine Rede gelangt (Rhet. Her. I 7, 11). Mit Dissimulatio im Sinne von Verstellung ist etwas anderes als die bereits besprochene Verbergung der eigenen Kunst gemeint. Insbesondere die Ironie, die «als das Gegenteil von dem, was ausgesprochen wird verstanden werden will» (Quint. inst. VI 2, 15), kann zu den Stilmitteln der Verstellung gezählt werden. Ironisch zu sprechen bedeutet, «in gespieltem Ernst des ganzen Stils der Rede» das Gegenteil von dem zu sagen, was man denkt (Cic. de orat. II 269). Cicero benennt die Stilfigur, «die etwas anderes meint, als sie sagt, und die gleichsam besonders tückisch in das Herz der Menschen schleicht» denn auch direkt als Dissimulatio bzw. Verstellung (Cic. de orat. III 203). Die Verstellung wirke besonders vorteilhaft, wenn sie «nicht mit engagierter Heftigkeit, sondern im Plauderton» vorgebracht werde (Cic. de orat. III 203), was eine Vertuschung der eigentlichen Absicht und Gestimmtheit des Redners bedeuten kann.

Mit ihrer Verhüllungstaktik, ihrem «So tun, als ob〉, kann die Ironie in zweifacher Weise einen Verstoß gegen die Wahrhaftigkeit verursachen: Bei der ironischen Dissimulatio kann es um die Verheimlichung der eigenen Denkvorgänge gehen, indem der Anschein erweckt wird, «man habe keine klare Vorstellung von dem, was der andere meint» (Quint. inst. VI 3, 85). In der stärkeren Form der Simulatio kann damit sogar vorgetäuscht werden, man habe eine bestimmte Überzeugung, die man jedoch gar nicht vertritt (Quint. inst. VI 3, 85). ${ }^{6}$ Anstelle einer Meinung können auch Affekte durch Verstellung simuliert werden, indem sich der Redner zürnend, ängstlich, erfreut oder erbittert gibt, obschon er nicht wirklich so fühlt. Diese Form der Simulatio dient Quintilian zufolge der Steigerung der Gefühlswirkung im Publikum (Quint. inst. IX 2, 26-27). Mittels Simulationsironie könne der Redner in dem, worin er sich der Gegenseite zu beugen scheint, auch einen «stillschweigenden» Vorwurf gegen sie kenntlich machen und damit den Hass noch stärker schüren als durch das direkte Vorgehen, alles frei herauszusagen (Quint. inst. VI 2, 16).

6 Die Unterscheidung zwischen der angeblich schwächeren Dissimulatio und der stärkeren Simulatio (vgl. Lausberg 1995: § 902, 446-447) wird in den klassischen Rhetoriktexten nicht konsequent gezogen. Zur Abgrenzung von der weiter oben ausgeführten Dissimulatio Artis verwende ich in diesem Abschnitt Simulatio als Oberbegriff für das Phänomen der Verstellung. 
Auch der entgegengesetzte Zweck lässt sich mittels Simulatio erreichen nämlich «die Mauer von Haß und Zorn» zu durchbrechen (Quint. inst. VI 3, 9). Wird die Verstellung nämlich in scherzhafter Weise eingesetzt, so kann sie für Amüsement und eine Auflockerung der Situation sorgen. Wie kein anderes Mittel der Rede helfe der ironische Witz laut Cicero dabei, «Bitterkeit und Strenge» zu mildern, die Situation zu entspannen und «Widerwärtigkeiten, denen mit Argumenten nicht leicht beizukommen ist, in Scherz und Lachen aufzulösen» (Cic. de orat. II 236). Belustigung kann laut Quintilian beispielsweise durch eine gezielte Doppeldeutigkeit, eine Übertreibung oder ein bewusstes Sichdummstellen entstehen (Quint. inst. VI 3, 85-95). Die Komik, die sich daraus ergibt, dass sich ein eigentlich verständiger Redner per simulationem als dumm hinstellt, wird auch bei Cicero erwähnt. Eine komische Situation lasse sich besonders gut erzeugen, wenn man eine Aussage absichtlich wörtlich nehme, anstatt auf das damit Gemeinte einzugehen (Cic. de orat. II 259). Oder wenn man «sich den Anschein gibt, etwas, das man versteht, nicht zu verstehen», woraus besonders ungereimte oder lächerliche Aussagen entstünden (Cic de orat. II 275). Überhaupt schreibt Cicero dem Humor des Redners eine große Kraft zu, weshalb er gar annimmt, dass besonders gewitzte Redner Richterstimmen gewinnen können durch ihren «beißenden, geistvollen und geschliffenen Witz und Humor» (Cic. de orat. I 243). Gerät eine Rede jedoch ungewollt lustig, würde man dies laut Quintilian auf die Dummheit (stultitas) des Redners zurückführen (Quint. inst. VIII 6, 74).

Die Ironie gilt schon Aristoteles als die Hauptform des feinen Witzes: «Der geistvolle Reiz eines Ausspruchs liegt darin, daß nicht gemeint ist, was gesagt wird» (Aristot. rhet. III 11, 6, 1412a). Er sieht die Verstellung nicht nur als Mittel der Einschmeichelung und Vergnügung, sondern auch der Aufmerksamkeitslenkung. Indem der Redner so tue, als gehe es um eine besonders bedeutende Angelegenheit, die das Publikum persönlich beträfe - oder gerade umgekehrt, als sei die behandelte Sache gänzlich unwichtig -, könne er die Aufmerksamkeit und Aufnahmebereitschaft des Publikums je nach Bedarf verstärken oder mindern (Aristot. rhet. III 14, 7, 1415a). Wolle man «keine aufmerksamen Hörer», solle man das behandelte Thema dem Publikum als «geringfügig, belanglos für sie und unerfreulich erscheinen lassen» (Aristot. rhet. III 14, 7, 1415b). Gerade ein unaufmerksames Publikum sei in gewissen Fällen wünschenswert: «Es ist ja keineswegs stets nützlich, wenn man den Zuhörer dazu veranlaßt, bei der Sache zu sein» (Aristot. rhet. III 14, 7, 1415a). Im Fall der Aufmerksamkeitsminderung verletzt der Redner allerdings nicht nur die Tugenden der Klarheit und Sachbezogenheit, sondern unterläuft zumindest vordergründig das von der Rhetorik angestrebte Ziel, die Aufmerksamkeit des Publikums zu gewinnen. Tatsächlich wird jedoch die Aufmerksamkeit des Publikums lediglich auf eine andere Ebene umgelenkt - wofür wiederum ein Witz besonders geeignet sein kann. Strate- 
gisch wird diese Form der Verstellung folglich als Ablenkungsmanöver eingesetzt. Dies gelingt laut Aristoteles vor allem bei wenig gebildeten Zuhörern: «Auf einen niveaulosen Zuhörer wirkt auch, wenn er etwas hört, was nicht zum Thema gehört» (Aristot. rhet. III 14, 8, 1415b). Aufmerksamkeitslenkung durch Vertuschung wird bei Cicero ebenfalls als Kunstmittel erwähnt: Nicht nur sollte der Redner gewisse Dinge bewusst übergehen oder übersehen (relinquat et neglegat), sondern manchmal solle er gar die Aufmerksamkeit vom relevanten Gegenstand ablenken (ab eo, quod agitur, avertat animos) (Cic. orat. 39-41, 134-39).

Etwas schwächer als die Simulationsironie, aber laut Quintilian eines der am häufigsten vorkommenden Stilmittel ist die verstellte Rede (oratio figurata), «bei der wir in einer Art von Argwohn das verstanden wissen wollen, was wir nicht sagen, nicht gerade das Gegenteil wie bei der Ironie, sondern etwas Verstecktes und dem Spürsinn des Hörers zum Suchen Überlassenes» (Quint. inst. IX 2, 65). Als Grund für diese Art der Verstellung wird genannt, dass es manchmal zu unsicher wäre oder sich nicht schicken würde, offen zu reden. Die Oratio figurata könne aber auch einzig um der schönen, neuen oder abwechslungsreichen Form willen gewählt werden (Quint. inst. IX 2, 66). Auch hier geht es im weitesten Sinn um Aufmerksamkeitslenkung und Einschmeichelung durch absichtliche Verstellung oder die Verschleierung des eigentlichen Sachverhalts. Im Extremfall kann es, so Quintilian, sogar angebracht sein, den Eindruck zu erwecken, man wolle sich am liebsten gar nicht äußern: «Alles in allem: der Richter schenkt dann den Figuren am meisten Vertrauen, wenn er meint, wir wollten überhaupt nicht reden» (Quint. inst. IX, 2, 72).

\subsubsection{Regelbruch und Anspielung: Das Spiel mit den Erwartungen des Publikums}

Die genannten Formen der Simulatio sind auch ein ideales Mittel zur Unterwanderung der Publikumserwartungen, um Überraschungs- und Belustigungsmomente zu kreieren. Die klassische Rhetorik widmet sich ausführlich dem Humor und der Abwechslung der Rede - und somit auch ihrem Unterhaltungsfaktor. Besonders zum Erzeugen von Lachern ist die Subversion der Erwartungen (praeter expectationem) des Publikums von großer Bedeutung, wie sowohl der Auctor ad Herennium (Rhet. Her. I 6,10) als auch Cicero festhalten: «Ihr wißt freilich, daß die bekannteste Form der Komik dann gegeben ist, wenn etwas anderes gesagt ist, als wir erwarten» (Cic. de orat. II 255; Beispiele Cic. de orat. II 284-285). Am leichtesten könne das Publikum gerade durch den eigenen Irrtum amüsiert werden (Cic. de orat. II 260). Durch das Täuschen der Publikumserwartungen kann der Redner einen geistreichen Eindruck hinterlassen und die allenfalls nachlassende Auf- 
merksamkeit des Publikums leicht wieder erlangen. Wenn das Publikum bereits ermüdet sei, könne unter anderem eine Vertauschung der Buchstaben - also ein eigentlicher Lapsus - die Zuhörer zum Lachen bringen oder überraschen (Rhet. Her. I 6, 10). Daneben können Zweideutigkeiten, die Entstellung von Wörtern, die Ähnlichkeit mit einem nur geringfügig veränderten Wort oder eine Übertreibung einen komischen Effekt haben (Rhet. Her. I 6, 10, Cic. de orat. II 249-250). Der Redner umgeht dabei gezielt Tugenden wie die Sprachrichtigkeit und Klarheit. Schon bei Aristoteles werden Überraschungseffekte genannt, die durch Sprachspiele zustande kommen, welche die grammatikalischen Regeln brechen - sei es durch spaßhaftes Wörterverdrehen, das Verwechseln von Buchstaben oder das Spiel mit Mehrdeutigkeiten, die als Lapsus oder Versprecher getarnt werden (Aristot. rhet. III 11, 6-7, 1412a). Oftmals wird dabei auf etwas angespielt, das nicht wörtlich gesagt ist: Die Entschlüsselung der Botschaft verlange dem Publikum einen kleinen Denkaufwand $\mathrm{ab}$, was besonders vergnüglich sei - jedoch nur dann, wenn rasch klar werde, was damit angedeutet wurde (Aristot. rhet. III 11, 6-7, 1412a).

\subsubsection{Authentizität und Selbstaffizierung: Als käme die Rede aus innerstem Herzen}

Bei all den genannten Verfahren der Dissimulatio und Verstellung darf eine Sache niemals vergessen werden: Sympathie und Glaubwürdigkeit kann ein Redner nur dann nachhaltig erlangen, wenn die in Rede und Vortrag vermittelte Persönlichkeit, die Emotionen und das Gesagte auf das Publikum echt wirken. Heutzutage würde man wohl sagen, dass es um die Authentizität des Redners gehe: «Aus rhetorischer Sicht ist Authentizität als performative Inszenierung von Echtheit, Natürlichkeit, Aufrichtigkeit, Originalität oder Unmittelbarkeit zu verstehen» (Ulrich 2012: 79). Das Verbergen der Künstlichkeit der Rede, das Erwecken von Spontaneität und Leichtigkeit, aber auch die Idee der bewusst rau belassenen Rede lassen sich unter diesem Aspekt des Authentischen betrachten. Gerade mit der Unverfälschtheit tritt ja die elaborierte Rede in Konflikt und je elaborierter sie ist, desto größer wird die Kluft zwischen dem Authentizitätsanspruch und der kunstvollen Ausarbeitung. Je mehr der Redner über die Wirkungsweisen von Ausdruck, Geste und Stimme, über die Schmuckmittel und Affektstrategien weiß und je bewusster er diese einzusetzen versteht, desto weniger wird sein «echter〉 Charakter, sein ursprünglicher Habitus nach außen durchdringen. In diesem Spannungsfeld bewegt sich die Rhetorik notgedrungen. Das Problem liegt auch im «paradoxalen Grundcharakter» der Authentizität: «sie ist, obwohl sie anders erscheint, immer eine hergestellte und damit sekundäre Echtheit [...]. Ihr Modus 
des 〈Als-ob〉 speist sich aus einer allgemeinen Sehnsucht nach primärer Echtheit, Natürlichkeit, Originalität oder Unmittelbarkeit, die immer dann entfacht wird, wenn eben diese Konzepte ins Wanken geraten» (Ulrich 2012: 80-81). Dieses Wanken wird umso spürbarer, je stärker sich menschliche Interaktion von ihrem (vermeintlichen) «Urzustand〉 in Richtung einer bewusst konzipierten Ausgestaltung von Kunst- und Kommunikationstechniken entwickelt.

Obgleich die antike Rhetorik dieses Paradox nicht explizit problematisiert, lassen sich bereits in der klassischen Rhetoriklehre ambivalente Aussagen bezüglich der Authentizität von Rede und Redner finden. Nachdem etwa der Auctor ad Herennium zunächst bis ins Detail die verschiedenen Möglichkeiten aufzählt, wie eine Rede gestisch und mimisch begleitet werden sollte, um sie mit Leidenschaft oder Würde, Ruhe oder Komik vorzutragen, gibt er schließlich zu bedenken: «Dies muss man jedoch wissen, daß ein guter Vortrag es bewirkt, daß der Anschein erweckt wird, die Rede komme aus dem innersten Herzen» (ut res ex animo agi videatur) (Rhet. Her. III 15, 27). Die emotionale Ergriffenheit des Redners wird somit zur «entscheidende[n] Voraussetzung dafür, daß eine Rede 〈authentisch〉 wirkt» (Till 2009: 1038). Die Doppelbödigkeit dieser Bedingung zeigt sich jedoch im Satz des Auctor ad Herennium, der besagt, die Rede solle so erscheinen, als käme sie aus dem Innersten. Die Rede soll wahrhaft authentisch wirken - aber kann und muss sie hierfür auch echt sein? Reicht es aus, wenn der Redner ergriffen wirkt oder muss er die zur Schau gestellten Gefühle auch tatsächlich empfinden?

Bei Cicero findet sich eine ähnliche Stelle, die zwischen der Forderung nach echter Emotion und bloß überzeugend vorgespieltem Gefühlsausdruck oszilliert. Es sei nämlich nicht möglich, im Zuhörer Gefühle des Hasses oder Mitleids $\mathrm{zu}$ evozieren, ohne dass diese dem Redner selbst «eingebrannt und eingeprägt erscheinen» (in ipso oratore impressi esse atque inusti videbuntur) (Cic. de orat. II 189). Auch hier wieder: Bedeutet dies, dass der Redner diese Gefühle wirklich haben muss, oder lediglich, dass er sie überzeugend vortäuschen können sollte? Tatsächlich sollte bei Cicero zumindest aus der Sicht des Antonius in diesem Punkt gerade nicht nach einer höheren Kunst der Verstellung oder des geheuchelten Gefühlsausdrucks gestrebt werden, sondern danach, diese Gefühle als Redner selbst zu empfinden: «Ich hätte bei Gott niemals vor Gericht mit meiner Rede Schmerz und Mitleid, Neid und Haß erregen mögen, ohne selbst bei der Beeinflussung der Richter von den Empfindungen bewegt zu werden, zu denen ich sie bringen wollte» (Cic. de orat. II 189). Ebenso berichtet Antonius aus einem Gerichtsfall, er habe «aus echter, schmerzlicher Bewegung» und keinesfalls «nach der Regel einer Kunst» die Tunika eines gramgebeugten Greises zerrissen, um dessen schlimme Narben zu zeigen (Cic. de orat. II 195). Wäre er selbst frei von Schmerz gewesen, schließt Antonius, hätte seine Rede nicht «mitleiderre- 
gend, sondern gar lächerlich gewirkt» (Cic. de orat. II 196). Nichtsdestoweniger bietet derselbe Antonius den Anwesenden an, sie darin zu unterweisen, «beim Reden Zorn und Schmerz zu fühlen und Tränen zu vergießen» (Cic. de orat. II 196). Die Selbstaffizierung des Redners erhält dadurch wiederum den Status einer Technik, die lernbar ist.

Quintilian leitet ebenfalls dazu an, die Gefühle selbst $\mathrm{zu}$ empfinden, $\mathrm{zu}$ denen man sein Publikum erregen möchte (Quint. inst. VI 2, 26-36): «Aber wie ist es möglich, sich ergreifen zu lassen? Die Gemütsbewegungen stehen doch nicht in unserer Gewalt!» (Quint. inst. VI 2, 28). Quintilian hält gerade dies für möglich und durch die eigene Praxis belegt: Man müsse lediglich seine Phantasie zu Hilfe nehmen und sich die Dinge, von denen man spricht, im Sinne der Evidentia gleichsam vor Augen führen (Quint. inst. VI 2, 29-32). Dabei solle man sich vorstellen, selbst betroffen zu sein, «als wären wir die vom Prozeß Bedrohten selbst» (Quint. inst. VI 2, 35). Aufgrund dieser Technik habe Quintilian selbst sich in Prozessen oftmals «so ergriffen gefühlt, daß es nicht nur Tränen bekundeten, die mich überkamen, sondern sogar Erblassen und ein solcher Schmerz, als wäre er echt» (Quint. inst. VI 2, 36). Selbst hier wird jedoch der Schmerz, den Quintilian als Redner empfindet, lediglich beschrieben als «dem wahren Schmerz ähnlich〉 (veri similis dolor). Kann der Gefühlsausdruck, den ein Redner durch den gezielten Einsatz der Methode der Selbstaffizierung erlangt, am Ende also wirklich authentisch genannt werden? Die Spannung zwischen der Kunst und der Möglichkeit nach «wahrer Echtheit〉 innerhalb derselben lässt sich niemals ganz auflösen.

\subsection{Die rhetorische Figur als Abweichungsmuster}

\subsubsection{Barbarismus und Solözismus: Der «feine Unterschied` zwischen Fehler und Figur}

Die Grammatiklehre innerhalb Quintilians «Ausbildung des Redners` nennt verschiedene Formen des sprachlichen Fehlers, die gegen die Tugend der Sprachrichtigkeit bzw. Fehlerlosigkeit der Rede (oratio emendata) verstoßen (Quint. inst. I 5, 1). Grob werden die Fehler eingeteilt in Barbarismen, welche sich in einzelnen Wörtern zeigen und in Solözismen, die aus der Zusammenstellung der Wörter bzw. der Syntax resultieren (Quint. inst. I 5, 6; 34). Ein Barbarismus kann beispielsweise durch die Auslassung von Buchstaben oder die Verwendung fremdsprachiger Ausdrücke zustande kommen, ein Solözismus durch eine Abänderung in der Wortfolge, eine Verwechslung der Tempora oder einen unvollständigen Satz (Quint. inst. I 5, 8-10; 38-41; IX, 3, 9-11; vgl. auch Aristot. rhet. III 5, 3-4, 1407a-b). 
Quintilian verwendet zur Kategorisierung dieser Grammatikfehler die sogenannte Quadripertita Ratio, ${ }^{7}$ bei der die Fehler aus vier Änderungskategorien hergeleitet werden: Aus der Erweiterung (adiectio), der Unterdrückung bzw. Weglassung (detractio), der Verwechslung (transmutatio) oder der Vertauschung (inmutatio) (Quint. inst. I 5, 6; 38-41). Grundsätzlich stellen all diese Formen der Veränderung aus Sicht der Grammatik einen Fehler und somit eine Form der Imperfektion dar. Gleichzeitig sind es aber exakt dieselben Möglichkeiten der Sprachgestaltung, die auch eine Vielzahl von rhetorischen Figuren hervorbringen. Bei Quintilian werden die Figuren denn auch definiert als Formen der vernünftigen oder wohlüberlegten Veränderung (cum ratione mutatio) des Sinns oder Ausdrucks gegenüber der gewöhnlichen, einfachen oder spontanen Erscheinungsform (Quint. inst. IX 1, 11; 13). Das Ziel aller Figuren der Veränderung «durch Vertauschung, Hinzufügung, Weglassen und Anordnung» sei es nicht nur, auf den Zuhörer anziehend zu wirken, ihn nicht müde werden zu lassen oder gar aufzuwecken, sondern diese Figuren «gewinnen einen eigenen Reiz aus ihrer Ähnlichkeit mit einem Ausdrucksfehler, wie bei Speisen zuweilen gerade das Saure den Genuß hebt» (Quint. inst. IX, 3, 27). Entsprechend kann exakt dieselbe sprachliche Form einmal als Fehler, einmal als Figur bewertet werden:

[M]anche Verbindungen haben das Ansehen eines Solözismus, können aber doch nicht als fehlerhaft bezeichnet werden [...]. Figuren [schemata] wird man sie also nennen, häufiger zwar bei den Dichtern, doch auch bei den Rednern gestattet. Eine echte Figur hat in der Regel einen vernünftigen Grund [habebit aliquam rationem] [...]. Doch auch was hier eben als Figur bezeichnet wird, wird, wenn es von jemandem aus Unüberlegtheit (per inprudentiam] gebildet wird, nicht frei vom Fehler des Solözismus sein. (Quint. inst. I 5, 52-53)

Die Entscheidung, einen grammatikwidrigen Ausdruck oder eine unzulässige Wortverbindung als Fehler oder als erlaubte Figur zu behandeln, wird bei Quintilian abhängig gemacht davon, ob sie vernünftig und mit Bedacht gewählt wurde, «denn jede derartige Figur wäre ein Fehler, wenn sie nicht gesucht würde, sondern unterliefe» (Quint. inst. IX 3, 2; ähnlich auch IX 31-35). Dies allerdings ist dem Gesagten äußerlich nicht anzusehen, sondern hängt von einer qualitativen Einschätzung ab. Dass die Grenzen zwischen Fehler und Tugend durchlässig sind, ist sich Quintilian bewusst: «Weil aber bisweilen diese Fehler entschuldigt werden durch den Sprachgebrauch, eine Autorität, Alter oder schließlich sogar durch die nachbarschaftliche Verbindung mit Vorzügen - denn oft ist es schwer, sie von den Figuren zu trennen -, muß der Grammatiker [...] seinen Scharfsinn auf den feinen Unterschied richten» (Quint. inst. I 5, 5; ähnlich auch IX 3, 3).

7 Wird oft als Quadripartita Ratio wiedergegeben. 
Um diese subtile Unterscheidung $\mathrm{zu}$ begründen und entsprechend eine Abweichung von der Norm entweder als Stilmittel zu legitimieren oder als Vitium $\mathrm{zu}$ verwerfen, kann man sich also auf die Autorität (auctoritas) bedeutender Autoren, Redner oder Historiker berufen (siehe auch Quint. inst. I 6, 42). Nicht alle Autoren werden dabei gleich streng bewertet - so seien etwa Dichter aufgrund des Verszwangs rascher entschuldigt als Redner (Quint. inst. I 5, 11f). Für die «zuverlässigste Sprachmeisterin» hält Quintilian jedoch den Sprachgebrauch (consuetudo), die «Üblichkeit der Ausdrucksmittel» (Quint. inst. I 5, 3). Die Kriterien der Autorität und des Sprachgebrauchs bedingen sich gegenseitig: Auch Autoritäten sollen an der zeitgemäßen Redeweise bemessen werden - gewisse Ausdrücke großer Autoren «könnte heute wohl niemand mehr ertragen» (Quint. inst. I 6, 42). Umgekehrt solle man nichts als Sprachregel nehmen, «wenn es sich in fehlerhafter Weise bei vielen festgesetzt hat», sondern es solle jener Sprachgebrauch als Referenz dienen, der unter Gebildeten Übereinstimmung finde (Quint. inst. I 6, 44-45). ${ }^{8}$

Mit dem Konzept der rhetorischen Figur schafft die klassische Rhetorik die Möglichkeit, grammatikalische Fehler als Kunstmittel zu legitimieren und diese von einer eigentlichen Imperfektion in eine Tugend der Rede umzudeuten. Die Unterscheidung von Fehler und Figur kann nicht anhand des Redetexts allein gezogen werden, sondern hängt erst einmal davon ab, ob ein sprachliches

8 Als weiteres Merkmal, das Barbarismen und Solözismen als zulässige Redemittel rechtfertigen könnte, ließe sich allenfalls noch die Nützlichkeit (utilitas) sehen. In Bezug auf den Ornatus weist Quintilian explizit darauf hin, dass gerade «auf diesem Gebiet Vorzüge und Fehler benachbart» seien (Quint. inst. VIII 3, 7). An der genannten Stelle begründet er die wahrhaft schöne und gepflegte Redeweise mit dem Nutzen und der Effizienz, die dadurch zugleich geschaffen würden, wobei er sie mit der Kultivierung des Landes vergleicht: «Sollte ich etwa ein Gut für gepflegter [cultior] halten, auf dem mir jemand Lilien, Veilchen, Anemonen und springende Quellen zeigt als eins, wo sich volle Ernte und von Trauben schwere Reben finden? [...] Zierlicher ist ein Pferd anzuschauen, dessen Flanken schlank sind, aber zugleich ist es auch schneller» (Quint. inst. VIII 3, 8-9). Wahre Schönheit wird mit Zweckmäßigkeit (utilitas) verbunden, und Schmuck sei weit mehr als bloßer Zierat (Quint. inst. VIII 3, 11). Eine antike Formulierung des modernen Prinzips «Form follows function` findet sich bei Cicero, wo sie gar einem teleologischen Naturprinzip zu entspringen scheint: «Doch wie es in so vielen Dingen die Natur selbst wunderbar gefügt hat, so fügt es sich auch in der Rede, daß das, was den größten Nutzen [utilitas] in sich trägt, zugleich am meisten Würde [dignitas] oder oft auch Schönheit [venustas] zeigt» (Cic. de orat. III 178). So sei die ganze Welt, die Natur, jeder Baum, der menschliche Körper «zum Heil und Wohlergehen aller» perfekt geformt und «gleichsam ein Kunstwerk, nicht ein Werk des Zufalls» (Cic. de orat. III 178-179). Umso mehr treffe es für hergestellte Dinge wie Schiffe oder Tempel, aber auch auf die Rede zu, «daß Nützlichkeit und fast Notwendigkeit eine gewisse Art von Reiz und Anmut im Gefolge hat» (Cic. de orat. III 178-181). Was also in der Rede seinen Zweck gut erfüllt, wäre nach diesem Verständnis auch das Richtige - und dieses kann mitunter eben in der Abweichung von der Regel liegen. 
Merkmal aus grammatikalischer oder rhetorischer Sicht beurteilt wird (vgl. Stockhammer 2005: 302). ${ }^{9}$ Indem eine rhetorische «Lizenz» vergeben wird, lassen sich Ausnahmen von der Regel und Verstöße gegen die Norm gleichsam «von höherer Warte aus (z.B. in poetischer Sprache oder für den Affektausdruck)» rechtfertigen (Hambsch 2009: Sp. 1146). Autorität im Sinne einer Deutungshoheit könnte hier - entgegen Quintilians Einschätzung - zum entscheidenden Kriterium werden: "Auctoritas is the person whose parole is forceful enough to change the langue» (Stockhammer 2005: 306).

Neben Vergehen gegen die Grammatik bzw. Sprachrichtigkeit können auch Verstöße gegen andere Tugenden der Rede als rhetorische Figuren gedeutet werden. Besonders häufig ist die Perspicuitas bzw. Verständlichkeit betroffen. Cicero zählt auf, was Redner beachten müssen, um ihre Rede klar und deutlich vorzubringen:

Natürlich durch korrektes Sprechen, durch Worte, die gebräuchlich sind und treffend das bezeichnen, was wir ausdrücken und erklären wollen, durch Vermeiden allzu langer Perioden und weit ausgesponnener Metaphern, die zum Vergleich aus anderen Bereichen übertragen werden, durch Verzicht auf die Zerstückelung von Sätzen, Verkehrung im Gebrauch der Zeiten, Verwirrung von Personen und Störung in der Reihenfolge. Wozu noch viele Worte? Die ganze Sache ist so einfach. (Cic. de orat. III 49)

Dass die Sache nicht ganz so einfach ist, zeigt sich darin, dass die klassische Rhetorik eine Vielzahl von Redeformen anerkennt, welche exakt gegen die von Cicero genannten Regeln der Klarheit verstoßen. So lassen etliche der bereits beschriebenen strukturellen Abweichungen im Redefluss, wie etwa die Abschweifung, die Aposiopese, oder Weisen der Verstellung wie die Ironie, eine Rede unklar, verwirrend oder umständlich werden. Dennoch werden sie nicht als zu vermeidende Fehler, sondern als zulässige oder gar wünschenswerte Figuren der Rede betrachtet. Im Folgenden soll entlang der vier klassischen Änderungskategorien genauer betrachtet werden, welche Abweichungen von den sprachlichen Normen als Imperfektionen im Sinne rhetorischer Figuren lizensiert werden und aus welchen

9 Robert Stockhammer (2005) versucht zu zeigen, dass Quintilian die «außerordentlich wirkungsmächtige Unterscheidung» zwischen einer grammatischen und einer rhetorischen Ebene deshalb eingeführt habe, weil er sich daran gestört habe, dass sich sprachliche Fehler bzw. Barbarismen und Figuren auf der Textebene nicht unterscheiden ließen (Stockhammer 2005: 302). Durch die Einführung dieser zwei Ebenen würden seither Abweichungen von der üblichen Redeweise auf der Ebene der Grammatik als Fehler gedeutet, auf der Ebene der Rhetorik jedoch als Tugend - und in chiastischer Umkehrung Nicht-Abweichung auf der rhetorischen Ebene als Fehler, auf der grammatischen hingegen als Tugend (Stockhammer 2005: 305). Je nachdem, welche Deutung gelegener komme, könne man sich also auf das Regelwerk der Grammatik oder der Rhetorik berufen, was mit der Gefahr von Willkür verbunden sei. 
Gründen dies geschieht bzw. zu welchen Zwecken diese Abweichungsmuster genutzt werden.

\subsubsection{Anapher, Tautologie, Pleonasmus: Wiederholung und Redundanz zur Bekräftigung und Affektsteigerung}

Die Erweiterung (adiectio) als fehlerhafte Abweichung kann entweder als Barbarismus auftreten, wenn etwa ein Buchstabe zu einem Wort hinzugefügt wird, oder als Solözismus, wenn ein Wort wiederholt oder die Rede durch Wörter ergänzt wird, die für die eigentliche Aussage unnötig sind (Quint. inst. I 5, 6; 38). Beim Auctor ad Herennium wird das Wiederholen von Worten in kurzer Folge grundsätzlich zum schlechten oder gar fehlerhaften Stil gezählt, da es gegen die Tugenden der Eleganz und der gehörigen Anordnung verstoße (Rhet. Her. IV, 17, XII). Aristoteles befindet, dass «die ständigen Wiederholungen in der griechischen Rede mit Recht mißbilligt werden» (Aristot. rhet. III 12, 2-3, 1413b). Dennoch seien gerade Wiederholungen im Zusammenspiel mit abwechselnden Wörtern ein wichtiger Bestandteil von Reden - ja die Wiederholung «bahnt der Redekunst gleichsam den Weg: «Dieser ist es, der euch bestohlen, dieser ist es, der euch getäuscht, dieser ist es, der euch zu guter Letzt noch zu verraten versucht hat!»» (Aristot. rhet. III 12, 2-3, 1413b). Durch die Wiederholung wird in Aristoteles' Beispiel die Spannung kurz und kompakt auf einen Höhepunkt hin gesteigert und somit eine Klimax erreicht. Die Wiederholung lasse sich auch dazu verwenden, dem Zuhörer einen Punkt gut einzuprägen (Aristot. rhet. III 19, 4, 1419b). Ähnliche Funktionen der Wiederholung werden von Demetrios erwähnt: Einerseits diene die Wiederholung einzelner Ausdrücke der Erzeugung einer erhabenen und kraftvollen Wirkung, andererseits der Verdeutlichung und Emphase, damit ein wichtiges Wort nicht einfach überhört werde (Demetr. eloc. III 140; IV 196-197).

Wiederholung lässt sich also vielfältig als Kunstmittel einsetzen und wird der oben genannten Fehlerkategorie entsprechend zu den Figuren der Hinzufügung (per adiectionem) gezählt (Quint. inst. IX 3, 28). Cicero fordert denn auch von einem kunstfertigen Redner, er solle mitunter das wiederholen, was er schon gesagt habe (Cic. orat. 40, 137) und zählt in rascher Folge verschiedene Figuren der Wiederholung auf. Unter den Gedankenfiguren findet sich bei ihm die Iteratio, was schlicht als «Wiederholung〉 übersetzt wird - gemeint ist vermutlich die Epanalepse (Wiederaufnahme eines Wortes) -, und unter den Ausdrucksfiguren werden gleich vier Wiederholungsformen erwähnt: Die Geminatio (unmittelbare Verdoppelung eines Wortes, Anadiplose), die Repetitio (Wortwiederholung am Anfang, Anapher), die Conversio (Wortwiederholung am Ende, Epipher) und die Complexio (Wortwiederholung am Anfang und Ende, Symploke) (Cic. de Orat. 
III 203; 206). Diese (teils anders benannten) sowie weitere Figuren der Wiederholung finden sich auch bei Quintilian und beim Auctor ad Herennium: Neben Geminatio, Repetitio, Conversio und Conplexio werden etwa Conduplicatio, Traductio, Tautologie sowie Epanalepse genannt (Quint. inst. IX 3, 28-29, 68-71; 97; VIII 3, 50, 88; Rhet. Her. IV 28, 38; 13, 19). ${ }^{10}$

Laut Auctor ad Herennium bewirkt der wiederholte Einsatz eines Wortes bei figuraler Verwendung nicht nur keinen Anstoß (non offendat animum), sondern sogar eine kunstvollere Anordnung der Rede (concinniorum orationem) - besonders wenn mit unterschiedlichen Bedeutungen des Wortes gespielt werde (Rhet. Her. IV 14, 20). In diesen Fällen geschehe es nicht «durch den Mangel an Wörtern, dass man öfter auf dasselbe Wort zurückkommt», sondern es liege «eine Anmut [festivitas] darin, die leichter durch die Ohren unterschieden als durch Worte gezeigt werden kann» (Rhet. Her. IV 14, 21). Ob ein Sprachmerkmal wie die Wiederholung als Figur oder als Fehler zu beurteilen ist, wird hier zu einer Sache des 〈Hinhörens〉. Es scheint auch hier keine benennbare Regel zu geben, welche äußere Merkmale für das Vorhandensein einer Wiederholungsfigur im Gegensatz zu einem Fehler angeben könnte. Dasselbe Phänomen, die Wiederholung in Form der Tautologie oder der Epanálēpsis, kann uns laut Quintilian einmal als Fehler erscheinen, ein andermal als Figur, als Vorzug des Stils, welcher die Einprägsamkeit erhöhe (Quint. inst. VIII 3, 50-51; IX 2, 4). Analog wird der Pleonasmus, welcher die Rede «mit überflüssigen Worten» belaste, in manchen Fällen als Fehler bewertet (z.B. im Satz: «Ich selbst habe es mit eigenen Augen gesehen»), in anderen jedoch als Stilmittel der Bekräftigung und Präzisierung genannt: «Jedoch ist dies [...] ein Fehler, wenn der Ausdruck durch überflüssige Zufügung überlastet wird, wenn es aber den Gedanken hebt und verdeutlicht [...], ein Stil-Vorzug: er hat es gesehen, er selbst, leibhaftig vor Augen: das sind ebenso viele Affektstufen (wie Worte)» (Quint. inst. IX 3, 46). Hier zeigt sich beispielhaft, wie durch eine pleonastische Wiederholung die Affektwirkung gesteigert werden kann. Mit der Wiederholung arbeitet, wie wir in Aristoteles' Beispiel weiter oben schon gesehen haben, denn auch die Klimax oder Gradatio: «sie wiederholt nämlich, was gesagt ist, und bevor sie zum Nächsten aufsteigt, verweilt sie beim Vorausgehenden» (Quint. inst. IX 3, 55). Auch das Verdoppeln von Verben (verba geminari) dient Quintilian zufolge dazu, die Rede eindringlicher (wörtlich: schärfer, acrius) zu gestalten und ihr Reiz (gratia) und Kräfte (vires) zu verleihen (Quint. inst. IX 3, 28). Damit lasse sich mal die würdevolle, mal die klagevolle Aussage vergrößern

10 Zusätzlich zu den simplen Formen der Wiederholung erwähnt Quintilian chiastisch oder verflochten angeordnete Wiederholungsmuster innerhalb der Satzkonstruktion (Quint. inst. IX 3, 30-40), die Wiederholung ganzer Satzteile (43-43) oder von Worten über den Satz hinaus (44). 
(amplificare). Bei der Geminatio diene lediglich das erste Wort der Mitteilung, das zweite dagegen ausschließlich der Bekräftigung (Quint. inst. IX 3, 28).

So unterschiedlich die Ausformungen der Wiederholung, Redundanz und Anhäufung sein mögen: "Ihre Quelle aber ist ganz einheitlich: denn sie macht alles, was wir sagen, energischer und eindringlicher und läßt eine Kraft zur Schau tragen, als käme immer wieder die Leidenschaft zum Durchbruch» (Quint. inst. IX 3, 54). Die Figuren der Wiederholung werden in der Rhetorik also deshalb nicht als Fehler verworfen, weil sie eine «über die bloße Informationsfunktion hinausgehende affektisch-vereindringlichende Funktion» haben (Lausberg 1990: 311, § 612). Kurz gesagt: «Die Wiederholung ist eine 〈Pathosformel»» (Lausberg 1990: 311, § 612).

\subsubsection{Weglassung, Ellipse und unvollständiger Ausdruck zur Erzeugung von Kürze und Spannung}

Ebenfalls in den Bereich des grammatikalischen und stilistischen Fehlers hinein reichen Formen der Weglassung (per detractionem) bzw. der Ellipse, «wenn nämlich zum vollständigen Ausdruck etwas fehlt» (Quint. inst. I 5, 40; VIII 3, 50). Jede unvollständige Formulierung läuft grundsätzlich den Tugenden der Sprachrichtigkeit und Klarheit zuwider, weshalb sie als Fehler zu vermeiden ist. Doch auch die Dectractio oder Ellipse kann, «wenn es mit Überlegung geschieht», zur Figur werden (Quint. inst. VIII 3, 50). Laut Quintilian dienen die Figuren der Weglassung (per detractionem fiunt figurae) der Erzeugung von Reiz vor allem «durch ihre Kürze und Neuheit» (Quint. inst. IX 3, 58). Dabei werde der unterdrückte Ausdruck zwar meist hinreichend durch die übrigen Worte verständlich gemacht, wie etwa in den Cicero entnommenen Beispielen: «Selbstverständlich kein Wort sonst, nur über dich!» oder «Morgen die Briefboten!» (Quint. inst. IX 3, 58), doch ergebe sich in den Beispielen durch die Weglassung eine interessante Spannung. Manchmal erfordere es auch die Schicklichkeit, dass ein unanständiges Wort ausgelassen werde (Quint. inst. IX 3, 59).

$\mathrm{Zu}$ den Figuren der Weglassung lässt sich auch die bereits eingeführte Aposiopese (Reticentia, Praecisio) rechnen, bei welcher die Rede plötzlich abgebrochen wird. Bei der Aposiopese entsteht nicht nur durch das unvermittelte Abreißen des Redeflusses eine Imperfektion, sondern auch durch die grammatikalische Unvollständigkeit, da das Verstummen für gewöhnlich eine Auslassung von Wörtern zur Folge hat, die zur Vervollständigung des Ausdrucks nötig gewesen wären. Dennoch hat das unvermittelte Verstummen seine klaren Vorzüge als Figur. Im Unterschied zur Wortfigur der Detractio, bei der einfach ein Wort oder Ausdruck fehle, das jedoch «deutlich greifbar» sei, werde bei der Gedankenfigur 
der Aposiopese etwas Ungewisses oder nur in längerer Rede Erklärbares bewusst verschwiegen (Quint. inst. IX 3, 60). Noch mehr als mit der einfachen Auslassung von Wörtern kann mit einem abrupten Redeabbruch eine intensive Spannung erzeugt oder die Aufmerksamkeit auf eine Sache gelenkt werden, ohne diese jedoch aussprechen zu müssen.

Schließlich kann die unverbundene Rede, wie wir sie weiter oben als Imperfektion im Redefluss kennengelernt haben, auch unter dem Aspekt der Detractio betrachtet werden: Die für den korrekten Satzbau benötigten Bindewörter werden hier bewusst weggelassen, um Wirkungen wie Emphase, Kürze und eine Affekterhöhung zu erreichen. Das Asyndeton bzw. die gezielte Weglassung von Konjunktionen gilt daher als Figur der «pathetisch-vereindringlichenden Steigerung» (Lausberg 1990: 353, § 709).

\subsubsection{Hyperbaton, Anastrophe: Umstellung und Abweichung von der vorgegebenen Abfolge im Sinne von Geschliffenheit und Timing}

Die Grammatik beschreibt klare syntaktische Regeln, die darüber bestimmen, wie die Wörter im Satz anzuordnen und miteinander zu verbinden sind. Abweichungen von der vorgegebenen Wortfügung, die durch eine Umstellung (inversio) oder Verwechslung (transmutatio) der Wörter entstehen, werden deshalb in der Regel als Solözismen und somit als Fehler behandelt (Quint. inst. I 5, 40). Abweichungen von der vorgegebenen Buchstabenfolge innerhalb eines Wortes gelten in analoger Weise als Barbarismen. Gerade solche Umstellungen der vorgegebenen Buchstabenfolge oder syntaktischen Struktur können jedoch auch zum Kunstmittel werden. Quintilian zufolge ist der Redner manchmal sogar dazu angewiesen, die Wortfolge, wie sie natürlicherweise oder der Sache entsprechend vorgegeben wäre, zu durchbrechen, also etwa ein Wort zu überspringen oder an eine andere Stelle zu versetzen (Quint. inst. VIII 6, 62). Die bivalente Rolle der Transmutatio zeigt sich darin, dass sich bei Quintilian beispielsweise das Hyperbaton (Satzumbau durch Einschub bzw. Auseinanderstellen zusammengehöriger Wörter oder Wortteile bzw. das «Überspringen eines Wortes») oder die Anastrophe (Inversion der Wortfolge, z.B. in Form des Hýsteron-Próteron, d.h. das eigentlich später folgende Wort vor das frühere stellen) sowohl unter den Solözismen als auch unter den Stilvorzügen und Schmuckmitteln finden lassen (Quint. inst. I 5, 40; VIII 6, 62; 65). Cicero erwähnt unter den Figuren des Ausdrucks sogar direkt die Transgressio, also die "Abweichung von der regelmäßigen Wortstellung» (verborum concinna transgressio) (Cic. de orat. III 207).

Unter welchen Umständen wird das regelwidrige Umdisponieren der Wortfügung zur rhetorischen Figur erklärt? Ohne die Stilmittel der Transmutatio würde 
Quintilian zufolge manche Rede holprig und ungepflegt wirken: «Denn nur zu oft wird die Rede rauh, hart, zerhackt und rissig, wenn die Worte so gestellt werden, wie es den Bedürfnissen ihrer eigenen Reihenfolge entspricht» (Quint. inst. VIII 6, 62). Um der Rede Geschmeidigkeit zu verleihen, müsse man notgedrungen von der naheliegenden Reihenfolge abweichen. Wörter, die den Redefluss stören, müssten «zurückgestellt und vorausgenommen werden» (Quint. inst. VIII 6, 63). Quintilian bedient sich an dieser Stelle erneut der Handwerksanalogie, bei der die Tätigkeiten des Behauens, Polierens und Schleifens herangezogen werden:

[J]edes Wort [muß] wie beim Aufschichten von unbehauenen Steinen an die Stelle gesetzt werden, wohin es paßt. Denn wir können sie ja nicht behauen [recidere] und glätten [polire], damit sie sich zusammengehäuft besser verbinden, sondern müssen sie nehmen, wie sie sind, und den passenden Platz für sie aussuchen. (Quint. inst. VIII 6, 63)

Gerade weil es dem Redner nicht möglich sei, an den Worten selbst zu schleifen, solle er dieses Defizit wettmachen und seine Kunstfertigkeit beweisen, indem eine Gesamtkonstruktion erschaffen werde, in der sich die vorhandenen «Wortbausteine` geschmeidig ineinanderfügen und die Rede einen schönen Klang erhalten würde (ähnlich auch Quint. inst. IX 4, 27). Die Abweichung von der Norm hat hier zum Ziel, den Elaborationsgrad im Sinne der Geschliffenheit und Harmonie der Rede zu steigern.

Auch in Bezug auf die Anordnung des Stoffes gibt es Situationen, welche eine bewusste Abweichung von der Regel erfordern - und zwar im Sinne des Timings. Im Normalfall sollte zwar, wie der Auctor ad Herennium sagt, die Anordnung gemäß der Kunstlehre ( $a b$ institutione artis) nach einem klar geregelten Ablauf ausgeführt werden. Doch stelle sich den Regeln der Kunst eine Anordnung gegenüber, die sich den Zeitumständen anpasse (ad casum temporis accommodatum) (Rhet. Her. III 9, 16). Die Umstände des Augenblicks könnten es nämlich erfordern, dass der Redner die vorgegebene Reihenfolge durchbrechen müsse, etwa «wenn die Ohren der Zuhörer schon völlig betäubt scheinen und ihre Sinne erschöpft» (Rhet. Her. III 9, 17). In diesem Fall könne durchaus ein vorgesehener Schritt ausgelassen werden, um stattdessen mit einer kraftvolleren Stelle zu beginnen - auf die früheren Passagen könne man ja später immer noch zurückkommen (Rhet. Her. III 9, 17). Auch bei schwierigen, emotional aufgeladenen Themen könne eine solche Abweichung erforderlich werden: «Diese Veränderungen und Umstellungen muß man oft notgedrungen vornehmen, wenn die Sache selbst dazu zwingt, die kunstvolle Anordnung kunstvoll zu verändern» (artificiosam dispositionem artificiose commutare) (Rhet. Her. III 10, 18). Der Regelbruch läuft in diesem Fall nicht eigentlich der Kunst entgegen, sondern ist deshalb eingefordert, weil zwei 
Kunstlehren bzw. Regelwerke aufeinanderprallen: jene der richtigen Reihenfolge und jene des richtigen Moments oder Kairós.

\subsubsection{Metapher, Übertragung und uneigentliche Rede als vielfältige Schmuckmittel der Veranschaulichung, Unterhaltung und Gewitztheit}

Eine Vielzahl stilistischer Möglichkeiten ergibt sich schließlich durch die Änderungskategorie der Vertauschung oder Ersetzung, Immutatio genannt. Auch die Vertauschung kann entweder als Fehler oder Figur gedeutet werden. Quintilian spricht von einer Inmutatio, «wenn das eine für ein anderes gesetzt wird» (Quint. inst. I 5, 41). Einerseits wird damit ein von der Transmutatio kaum zu unterscheidendes Phänomen benannt, das als Figur seinen eigenen Reiz entwickeln kann. Etwa wenn Cicero von den Ausdrucksfiguren einer «kleinen Änderung» eines Wortes (paulum immutatum verbum) oder der Vertauschung oder Ersetzung von Ausdrücken (immutatio) innerhalb der Rede spricht (Cic. de orat. III 206-207). Andererseits gehört zu den Formen der Vertauschung aber auch jene ganz besondere Weise der Veränderung, die Quintilian als Trópos bezeichnet. Während die normale Figur (figura) ganz einfach als eine Redeform betrachtet wird, «die abweicht von der allgemeinen und sich zunächst anbietenden Art und Weise», ist mit Trópos eine Redeweise gemeint, «die von ihrer natürlichen und ursprünglichen Bedeutung auf eine andere übertragen ist», in welcher also «Wörter für andere Wörter gesetzt» werden (Quint. inst. IX 1, 4). Zu den Tropen zählt Quintilian eine Vielzahl von Stilmitteln, insbesondere die Metapher (von griech. metaphorá: Übertragung), sowie die Metonymie, Antonomasie, Metalepse, Synekdoche, Katachrese, Allegorie, Onomatopoiie, Hyperbel, Periphrase, das Epitheton und das Hyperbaton (Quint. inst. IX 1, 5). ${ }^{11}$

Durch die Übertragung (translatio) der eigentlichen Bedeutung eines Ausdrucks auf einen neuen Sinnzusammenhang entsteht die uneigentliche Rede. Formen der uneigentlichen Rede sind insofern als Imperfektionen zu betrachten, als sie wortwörtlich genommen falsch, unwahrscheinlich, sinnlos oder unverständlich sind. Sie widersprechen somit dem Gebot der Wahrheit bzw. Wahrscheinlichkeit und der Forderung nach Durchsichtigkeit. Betrachtet man die Aussage jedoch unter dem Aspekt der Immutatio und folgt der mit ihr verbun-

11 Laut Quintilians Definition sind die Tropen von den Figuren zu unterscheiden, «denn eine Figur kann mit Worten in ihrer eigentlichen Bedeutung und Wortstellung zustande kommen», was für die Tropen gerade nicht gilt (Quint. inst. XI 1, 7). Da diese Unterscheidung aber selbst innerhalb der klassischen Rhetorik oftmals nicht konsequent berücksichtigt wird und auch Tropen oftmals als Figuren bezeichnet werden, subsumiere ich die Tropen hier unter die Figuren. 
denen Übertragungsleistung, lässt sich erfassen, was mit dem Gesagten gemeint wurde, wodurch die Aussage wieder korrekt und verständlich wird. Da nicht geradeheraus, sondern über Umwege gesprochen wird, wird hier aber immer noch ein im Grunde unnötiger Denkaufwand vom Publikum verlangt. Quintilian deutet die Abweichung von der eigentlichen Rede denn auch als Fehler, der generell nur dort zulässig sei, wo keine ursprüngliche Bezeichnung zur Verfügung stehe (Quint. inst. VIII 2-4). Gleichwohl hält er gerade die Translatio bzw. den übertragenen Gebrauch für jenen Abweichungsmodus, «in dem ja wohl der wichtigste Schmuck der Rede besteht.» Die Translatio mache «Worte für Dinge passend, die es eigentlich nicht sind» (Quint. inst. VIII 2, 6). Die übertragene Rede wird also nicht nur dort eingesetzt, wo die Worte fehlen, sondern dient, wie wir bei Cicero sehen, der Verwirklichung mannigfaltiger Wirkungsintentionen:

[D]ort, wo eigene Ausdrücke in Fülle zu Gebote stehen, gefallen trotzdem die uneigentlichen, wenn ihre Übertragung wohlberechnet ist, den Leuten noch viel mehr. Das mag entweder daher rühren, daß es ein gewisses Zeichen von Genie ist, das zu übergehen, was einem vor den Füßen liegt, und etwas anderes zu nehmen, was weit hergeholt ist; oder es kommt davon, daß der Zuhörer in Gedanken in eine andere Richtung geführt wird, ohne freilich von dem rechten Wege abzuirren, was besonders reizvoll ist; oder der Grund besteht darin, daß ein Wort jeweils eine Wirklichkeit entstehen läßt, oder es liegt daran, daß jede Übertragung, die man mit Verstand [ratione] vornimmt, unmittelbar die Sinne anspricht, vor allem den Gesichtssinn, der besonders lebhaft reagiert. (Cic. de orat. III 159-160)

Wird die uneigentliche oder übertragene Rede gekonnt und - was wir bereits mehrfach als Unterscheidungsmerkmal zwischen Fehler und Figur gesehen haben - mit Berechnung angewendet, kann der Redner damit seine eigene Brillanz hervorheben, die Zuhörer auf eine Gedankenreise mitnehmen und sie auf der sinnlichen Ebene berühren. Der Auctor ad Herennium fordert explizit ein, dass die Translatio sowohl bescheiden (pudens) als auch überlegt (cum ratione) eingesetzt werden müsse, damit «nicht der Eindruck entsteht, sie sei ohne Wahl unüberlegt und gierig auf ein unähnliches Gebiet übergesprungen» (Rhet. Her. IV 34, 45). Sind diese Voraussetzungen erfüllt, betrachtet der anonyme Autor die Translatio ebenfalls als geeignetes Mittel, um dem Publikum einen Sachverhalt vor Augen zu führen, diesen kurz und bündig darzustellen und die Rede zu schmücken (Rhet. Her. IV 34, 45). Im Sinne des Ornatus und seiner vielfältigen Wirkungen lassen sich die Verstöße gegen Wahrscheinlichkeit und Durchsichtigkeit somit legitimieren (Quint. inst. VIII 3, 15).

Bei der Hyperbel ist die Verletzung des Wahrheits- bzw. Wahrscheinlichkeitsgebots am offensichtlichsten, da dieser Fehler ein notwendiger Bestandteil für das Vorkommen der Figur ist. Aristoteles führt die Hyperbel als eine Form der Metapher bzw. Übertragung ein, «wenn man etwa über einen, dem ein blaues 
Auge verpaßt wurde, sagt: 〈Ihr hättet geglaubt, er sei ein Maulbeerkorb»» (Aristot. rhet. III 11, 15, 1413a). Was in diesem Beispiel in übertreibender Absicht gesagt wird, ist offensichtlich falsch. Dennoch lässt sich leicht verstehen, was damit gemeint ist. Eine gekonnte Übertreibung kann, so Quintilian, dem Witz (urbanitas) der Rede dienen und in positivem Sinn für Gelächter sorgen (Quint. inst. VIII 6, 74). Gemäß Aristoteles kann die Hyperbel eine Heftigkeit offenbaren, wie sie jungen Männern oder Erzürnten am ehesten zustehe (Aristot. rhet. III 11, 15, 1413a). In der Charakterisierung durch den Auctor ad Herennium ist die Supralatio wesentlich durch die Imperfektion geprägt, da sie sich «über die Wahrheit hinwegsetzt, um etwas zu steigern oder abzuschwächen» (Rhet. Her. IV 33, 44). Auch Quintilian zufolge wird bei einer Hyperbel mehr gesagt, als geschehen sei, weshalb sie in jedem Fall über das Glaubhafte hinausgehe (ultra fidem) (Quint. inst. VIII 6, 67-68; 73). Cicero benennt die Figur gar direkt als «Übersteigerung der Wahrheit» (veritatis supralatio), die dazu eingesetzt werde, eine Sache zu vergrößern oder zu verkleinern (Cic. de orat. III 203). Obschon die Hyperbel per definitionem einen Verstoß gegen die Wahrheit oder zumindest gegen das Glaubliche beinhaltet, wird auch hier eine Grenze zwischen einem tolerierbaren Vergehen und dem zu verwerfenden Vitium gezogen. Quintilian zufolge kann der Fehler der Lüge nur dann zu einer Tugend werden, wenn man durch die Lüge nicht betrügen wolle und wenn die Übersteigerung des Glaubhaften nicht maßlos sei, wodurch sie der Künstelei (kakozēlía) anheimfiele (Quint. inst. VIII 6, 73-74).

\subsection{Stilhöhe: Imperfektion zur Regulierung der Intensität}

Ausgewogenheit, Schönheit und Wohlklang der gewählten Worte sind ein wichtiges Anzeichen für die elaborierte Rede. Deshalb werden Abweichungen von der harmonischen Komposition und vom ausgewogenen Klangbild, wie etwa das Zusammenprallen von Vokalen (Hiat) in aufeinanderfolgenden Wörtern oder auch eine abgehackte oder raue Stimme, im Rahmen der klassischen Rhetorik normalerweise als Fehler gedeutet. Zur Intensivierung oder Abschwächung der Intensität einer Rede kann es jedoch nötig werden, bewusst von den Vorgaben der harmonischen oder schmuckvollen Elaboration oder der erhabenen Stilhöhe abzuweichen. Gerade die Stimme des Orators ist für Cicero wie ein Instrument, das zum Ausdruck und Evozieren ganz unterschiedlicher Affekte in ihrer ganzen Vielfalt genutzt werden solle: «sanft oder rauh, gepreßt oder verströmend, getragen oder abgehackt, dumpf oder kreischend, mit wechselndem Ton an- oder abschwellend» (Cic. de orat. III 216). Alle diese Stimmvariationen lassen sich laut Cicero kunstvoll verwenden, ob sie nun wohlklingend oder dissonant, wirkungs- 
voll oder dezent sind: «Sie bieten sich dem Redner, wie dem Maler seine Farben, zur Abwechslung an» (Cic. de orat. III 217).

\subsubsection{Hervorbrechendes Pathos, Kakophonie und Hässlichkeit: Die unverfälscht intensive Wirkung des Disharmonischen}

Geht es um den Ausdruck von Gefühlen, sollten Klang und Fluss der Rede die Eigenart dieser Gefühle annehmen. Dem Aptum entsprechend müsse daher, wie Cicero betont, eine jähzornige Rede scharf und erregt klingen und immer wieder abbrechen, eine Trauerrede klangvoll, stockend und klagend, Furcht solle sich durch eine gedämpfte, unsichere und entmutigte Stimme ausdrücken, Kraft mit Schwung, Ungestüm und Nachdruck im Klang, Freude mit einer fließend zarten, fröhlichen und entspannten Stimme (Cic. de orat. III 217-219). Wie Aristoteles ausführt, kann die emotionale Erregung des Redners und der Zuhörer eine normalerweise schwülstig oder pathetisch wirkende Ausdrucksweise rechtfertigen, wie etwa die überschwängliche Verwendung von Epitheta, Fremdwörtern oder zusammengesetzten Wörtern: «Denn wenn man in Begeisterung versetzt ist, läßt man solche Äußerungen fallen, so daß auch die Zuhörer das ganz selbstverständlich in sich aufnehmen, weil sie in ähnlicher Stimmung sind» (Aristot. rhet III 7, 11, 1408b). Das offensichtliche Pathos wirkt in diesem Fall authentisch. Quintilian verlangt im Sinne des Ornatus zwar im Allgemeinen nach Wörtern und Wortverbindungen, die gegenüber anderen «anständiger, erhabener, glänzender, lieblicher und klangvoller» sind. Doch will er ihren Gebrauch ebenfalls an das Aptum angepasst sehen, weshalb für «Dinge, die schrecklich sind», auch «Wörter, die sich rauh anhören, besser passen» (Quint. inst. VIII 3, 17). Eine rohe, gleichsam natürlich hervorbrechende Art zu reden kann in bestimmten Fällen nicht nur passender, sondern auch wirkungsmächtiger sein als eine sorgfältig geschliffene Rede, wie Demetrios hervorhebt:

In Wahrheit wird nicht das vorher ausgeklügelte, sondern das von selbst entstandene Wort tiefen Eindruck hervorbringen, besonders wenn wir uns persönlich als erzürnt oder als geschädigt zeigen. Die Sorgfalt um die Glätte und Harmonie jedoch findet sich nicht bei einem zornigen Mann, vielmehr bei einem, der gefällig reden und noch mehr bei einem, der einen Festvortrag halten will (Demetr. eloc. V 300).

Der Gefühlsausdruck kann also authentischer und wuchtiger wirken, wenn er sich ungebremst in der Rede entlädt, als wenn sich darin ein sorgfältiges Bemühen um geordneten und wohlklingenden Ausdruck zeigt. Laut Demetrios können darum auch offensichtliche Fehler oder Unschönheiten wie der Hiat und das Asyndeton eine Rede mächtiger erscheinen lassen (vgl. Demetr. eloc. V 269, 299), und 
Missklang (kakophonía, dysphonía) lasse sich als kraftvolles Stilmittel einsetzen. Nur durch falsch klingende Worte lasse sich etwas Widerliches oder Schroffes mit genügend Anschaulichkeit (enárgeia) beschreiben (Demetr. eloc. IV 211) und erst die Disharmonie lasse eine Rede wirklich großartig und erhaben erscheinen: «Denn die Glätte und der Wohlklang haben ganz und gar keinen Platz im erhabenen Stil» (Demetr. eloc. II 48). ${ }^{12}$ Entsprechend meide etwa Thukydides die geschliffene und einheitliche Komposition, «ja er gleicht eher einem, der etwas holprig beim Gehen aufschlägt, wie die Wandrer auf den rauhen Wegen» (Demetr. eloc. II 48).

Eine eigentliche Zuwendung zum Hässlichen ist mit der Lizenzierung roher und falsch klingender Worte jedoch nicht ausgedrückt. ${ }^{13}$ Zwar spielen Hässlichkeit und Deformation insbesondere bei der Erzeugung von Komik eine wichtige Rolle (Cic. de orat. II 248): «Der Ort und gleichsam das Gebiet des Lächerlichen [ridiculus] [...] ist wesentlich bestimmt von einer gewissen Häßlichkeit [turpitudo] und Mißgestalt [deformitas]» (Cic. de orat. II 236; siehe auch Quint. inst. VI 3, 8). Doch auch wenn man «ausschließlich oder ganz besonders» über das Hässliche lache, so bedeute dies keinesfalls, dass das Hässliche auf hässliche Weise bezeichnet oder beschrieben werden solle (Cic. de orat. II 236). Im Gegenteil: Die Rede selbst dürfe sich nie durch Hässlichkeit auszeichnen. So hält Cicero es bei der übertragenen Rede für unangebracht, Gleichnisse herzustellen, die einen hässlichen, formlosen oder abstoßenden Eindruck erwecken könnten, und dies selbst dann, wenn zwischen Darstellung und Dargestelltem eine große Ähnlichkeit bestünde (Cic. de orat. III 163-164). In ähnlicher Absicht verbietet Quintilian alle Arten von hässlich klingenden, unanständigen, peinlichen oder obszönen Redeweisen und verurteilt sie als Fehler (Quint. inst. VIII 3, 44-47). Neben dem Hässlichen (turpitudo, deformitas, kakémphaton) sei auch das Niedrige (humilitas, tapeínosis) in der Rede zu meiden (Quint. inst. VIII 3, 48), und «für schmutzige Worte ist in einer gebildeten Rede überhaupt kein Platz» (Quint. inst. VIII 3, 17). Ähnlich wie die Figur ist der Einsatz disharmonischer oder hässlicher Elemente somit als kalkulierter Verstoß zu verstehen, der bei einzelnen Rednern

12 Rauheit in den Worten und in der Komposition empfiehlt Demetrios offenbar sogar zur Erzeugung von Erhabenheit (Demetr. eloc. II 49). Damit weicht er von klassischen Definitionen ab, die Erhabenheit mit Eleganz und Harmonie, Geschliffenheit und Perfektion und also mit einer stark elaborierten Form verbinden.

13 Dass beim Publikum schon früh eine Faszination für das Hässliche in der Kunst bestand, die jedoch aus Sicht der lateinischen Rhetoriker in der Rede nicht bedient werden sollte, lässt sich in der 〈Poetik〉 des Aristoteles sehen: «Denn von Dingen, die wir in der Wirklichkeit nur ungern erblicken, sehen wir mit Freude möglichst getreue Abbildungen, z.B. Darstellungen von äußerst unansehnlichen Tieren und von Leichen» (Aristot. poet. 4, 1448b). 
oder in besonderen Situationen dosiert eingesetzt wird, um zur Elaboration der Rede beizutragen.

\subsubsection{Verhaltener Stil: Die bewusste Herabsenkung der Intensität}

Möchte ein Redner dem Publikum seine Umgänglichkeit und Bescheidenheit ausdrücken, was wir ja als hilfreiche Ethos-Wirkung zum Gewinnen des Publikums herausgestellt haben, sollte der Redner auf ein allzu leidenschaftliches Gebaren verzichten: «Nicht immer ist ja ein energischer Charakter der Rede angezeigt, vielmehr ist es oft ein gefälliger, verhaltener und sanfter Stil, der die Betroffenen am ehesten empfiehlt» (Cic. de orat. II 183). Ein zurückhaltender Stil kann im Sinne der Dissimulatio Artis angebracht sein, um die Elaboriertheit der Rede unsichtbar zu machen und sich als Redner unauffällig und unverdächtig zu geben. Insbesondere für die Einleitung der Rede empfiehlt der Auctor ad Herennium deshalb, die Sprache «schlicht und einfach» zu gestalten und den «Gebrauch der Wörter gewöhnlich» zu halten (Rhet. Her. I 7, 11). Die Stilhöhe wird bewusst tief angesetzt, um nicht den Verdacht der kunstvollen Vorbereitung zu erwecken oder den Redner überheblich oder unsympathisch erscheinen zu lassen.

Der Verzicht auf intensive Stilmittel in der Rede kann auch den Zweck haben, eine Übersättigung des Publikums durch übermäßige Redeeffekte zu vermeiden. Wie Cicero bemerkt, würden wir gerade derjenigen Dinge am schnellsten überdrüssig, «deren Reiz unseren Sinnen am meisten Lust verschafft und deren erster Anblick am meisten Eindruck macht» (Cic. de orat. III 98). So seien leuchtende Farben, Falsett-Klänge oder intensive Parfums zwar viel reizvoller als unscheinbare Farben, klare, strenge Töne oder dezente Düfte, doch nutze sich unsere Freude daran rasch ab. Selbst die verlockendste süße Speise führe in kurzer Zeit zu einer Übersättigung. Es sei auch gar nicht möglich, den Sinnen einen ununterbrochenen Genuss zu verschaffen (Cic. de orat. III 99-100). Statt also stets nach den größten Wonnen für das Publikum zu streben, solle der Redner nach Worten suchen, welche die Sinne nur leicht erfreuten. Cicero empfiehlt einen dezenten, verhaltenen Stil, der inmitten heller Farben und glänzend geschmückter Worte für eine Pause oder für Abwechslung sorge, ein Stil, der die Rede nicht konstant steigere, sondern auch sinken und abklingen lasse (Cic. de orat. III 100-101): «Wir wollen also einen Redner haben, der zwar eindrucksvoll und angenehm wirkt - anders geht es eben nicht -, doch eine strenge und gediegene, nicht süßliche 
und abgeschmackte Anziehungskraft besitzt» (ut suavitatem habeat austeram et solidam, non dulcem atque decoctam) (Cic. de orat. III 103). ${ }^{14}$

Am Beispiel des Crassus zeigt Cicero auf, dass ein dezenter Redestil keineswegs weniger Anklang finden müsse als die erhöhte Stillage: «[S]chon seit langem sprichst du viel verhaltener und ruhiger, als du gewöhnlich sprachst. Und trotzdem findet deine ruhige Gelassenheit deiner so eindrucksvollen Redeweise jetzt nicht weniger Beifall, als damals deine außerordentliche Kraft und Energie gefunden hat» (Cic. de orat. I 255). Auch andere große Redner hätten es mit nur moderaten Steigerungen des Stils ihrer Rede erreicht, alle möglichen Wirkungen zu erzeugen. Außerdem hätten sie so vermieden, ihre «Lunge oder Stimme» unnötig zu strapazieren (Cic. de orat. I 255). Quintilian hebt sogar die Möglichkeit der Wirkungssteigerung durch eine Senkung der Stilhöhe hervor: «Auch gilt es ja nicht immer den Ton der Rede zu steigern, sondern auch, ihn zu senken. Gerade der niedrige Ton der Worte gibt zuweilen dem Gedanken seine Kraft» (Quint. inst. VIII 3, 21). Wenn also Cicero sich in seiner Anklage eines niedrigen, umgangssprachlichen Ausdrucks bedient habe, so bedeute dies nicht, dass ihm «ein ordinäres Wort» entfahren sei, vielmehr habe er damit «die Verachtung für den Menschen, den er vernichtet sehen wollte», noch gesteigert (Quint inst. VIII 3, 21).

\subsection{Fazit}

Wir haben zahlreiche Strategien der Imperfektion kennengelernt, die sich im System der klassischen Rhetorik trotz des antiken Perfektionsideals, der Orientierung an Ausgewogenheit und Harmonie und entgegen den geltenden grammatikalischen und stilistischen Normen durchsetzen konnten. Diese Imperfektionen haben ihre Vorzüge im Schmuck, in der Wirkkraft oder in ihrer affektiven und psychologischen Funktion: Sie emotionalisieren oder veranschaulichen eine Rede, wecken die Aufmerksamkeit des Publikums oder empfehlen den Redner.

Im Sinne der Naturbelassenheit der Rede wird empfohlen, gezielt Rauheit in den Redefluss zu bringen. Sowohl das abrupte Verstummen (Aposiopese), der unvermittelte Konstruktionswechsel (Anakoluth) als auch die unverbundene Aneinanderreihung, welche strikt genommen $\mathrm{zu}$ einem Abreißen (Abruptus)

14 Cicero bleibt hier eigentlich beim Vergleich mit der Speise, denn Suavitas heißt auch 〈Süße〉 oder «Wohlgeschmack〉 und die Adjektive austerus bzw. dulcis benennen die Geschmackseindrücke herb/bitter bzw. süß. Außerdem meint decoctus wörtlich «reif». Der Satz ließe sich entsprechend übersetzen als: «Wir wollen also einen Redner haben, der zwar eindrücklich und geschmackvoll wirkt - anders geht es eben nicht -, doch einen herben und festen, nicht süßlichen oder (über)reifen Wohlgeschmack besitzt.» 
des Redeflusses führen und somit regelwidrig die syntaktische Struktur und den Wohlklang der Rede stören, lassen sich als gezielte Brüche und dramaturgischer Kunstgriff verwenden, um die Rede eindringlicher, abwechslungsreicher und emotionaler $\mathrm{zu}$ gestalten. Abweichungen vom regelgemäßen oder aktuell gültigen Sprachgebrauch können die Wirkung des Gesagten verstärken und dem Redner Autorität verleihen. Auch was generell als überflüssig oder ungenau zu verwerfen ist, nämlich die ausschweifende oder umschreibende Rede, kann dort sinnvoll werden, wo die Aufmerksamkeit des Publikums von einem wenig rühmlichen oder unsittlichen Aspekt abgelenkt oder dieses in einen bestimmten emotionalen Zustand versetzt werden soll.

Im Sinne der Glaubwürdigkeit des Redners ist ein zurückhaltender Auftritt, ja gegebenenfalls ein bewusstes Understatement bezüglich der eigenen rednerischen Möglichkeiten ratsam, um Bescheidenheit und Redlichkeit $\mathrm{zu}$ bezeugen. Was primär als Ausdruck echter Zurückhaltung und Beschämung propagiert wird, lässt sich auch gezielt als Strategie der Dissimulatio Artis einsetzen. Dabei geht es einerseits um die Anwendung der Kunst bei gleichzeitigem Verbergen der durch verschiedene Elaborationsmodi und -schritte hervorgebrachten Künstlichkeit. Indem der Redner so tut, als geschehe alles ohne Anstrengung, Vorbereitung oder gar ohne großes Können, weist er den Verdacht der Künstelei, Prahlerei oder List von sich. Andererseits geht es um die verbale Herabsetzung des eigenen Könnens durch den Redner selbst - also im Grunde um eine zur Schau gestellte oder simulierte Imperfektion. Zur Redekunst, die sich kunstvoll verbirgt, um Vertrauen und Sympathien des Publikums zu gewinnen, gehört auch eine gewisse (echte oder scheinbare) Nachlässigkeit oder Nonchalance sowie der Anschein von Spontaneität und Leichtigkeit beim Sprechen. Wendet ein Redner die Simulatio oder Verstellung in der Rede an, begeht er bewusst einen Verstoß gegen die Wahrhaftigkeit oder gegen das Relevante, um sich geistreich beim Publikum einzuschmeicheln, Affekte zu schüren oder die Aufmerksamkeit von einem Sachverhalt abzulenken. Ein wichtiger Effekt der Simulationsironie ist auch das Brechen der Publikumserwartungen, um für Überraschung, Witz und Abwechslung zu sorgen. Um das Erwartbare zu umgehen und Anspielungen zu kreieren, bedienen sich Redner oftmals einer bewusst fehlerhaften Ausgestaltung der Rede und punktueller grammatikalischer Regelbrüche. Im Zentrum steht bei den Strategien der Dissimulatio die Authentizität des Redners.

Brechen sich Gedanken und Gefühle scheinbar ungefiltert Bahn, werden sie vom Publikum am ehesten für echt gehalten. Ein gewisser Überschwang, der eine ungeschliffene, disharmonische oder raue Sprache mit sich bringt, kann bei entsprechenden Gegenständen und Gefühlsregungen des Redners durchaus angemessen sein und zu einer intensiveren, affektgeladenen Wirkung führen. Umgekehrt kann eine gesenkte und verhaltene Stilhöhe die Redlichkeit und 
Zurückhaltung des Redners hervorheben. Nicht zuletzt erzeugen die rhetorischen Figuren - von der 〈Königsfigur` Metapher bzw. Übertragung zu den vielen anderen Formen der Veränderung und Abweichung von der Sprachnorm - zwar einen Verstoß gegen Sprachrichtigkeit, Wahrheit oder Deutlichkeit. Sie vermögen aber gerade aufgrund ihrer Abweichungsmuster (Hinzufügung, Weglassung, Verwechslung oder Vertauschung) Evidenz und Kürze, Witz und Brillanz, Freude und Schönheit zu bewirken und dienen damit wiederum der Elaboration der Rede.

Folgende Wirkungsintentionen lassen sich aus Perspektive der klassischen Rhetorik mit einer gezielten Imperfektion verfolgen:

Redner (Ethos / conciliare)

- Glaubwürdigkeit

- Wahrhaftigkeit

- Redlichkeit

- Bescheidenheit

- Authentizität

- Lockerheit

- Persönlichkeit

- Engagement

- Betroffenheit

Rede, Stilhöhe (Logos / docere; delectare)

- Vehemenz

- Prägnanz

- Eindringlichkeit

- Anschaulichkeit

- Kraft

- Emotionalität

- Verständlichkeit

Publikum (Pathos / movere)

- Sympathie

- Wohlwollen

- Aufmerksamkeit

- Interesse

- Affekte

- Abwechslung 